\title{
Design and Analysis of a Hierarchical IP Traceback System
}

\author{
by
}

\author{
Abes Dabir, B.A.Sc. \\ A thesis submitted to the \\ Faculty of Graduate Studies and Research \\ in partial fulfillment of the requirements for the degree of
}

\section{Master of Applied Science in Electrical Engineering}

Ottawa-Carleton Institute for Electrical and Computer Engineering

Department of Systems and Computer Engineering

Carleton University

Ottawa, Ontario

January 15, 2009

(C)Abes Dabir, 2009 


$\begin{array}{ll}\begin{array}{l}\text { Library and } \\ \text { Archives Canada }\end{array} & \begin{array}{l}\text { Bibliothèque et } \\ \text { Archives Canada }\end{array} \\ \begin{array}{l}\text { Published Heritage } \\ \text { Branch }\end{array} & \begin{array}{l}\text { Direction du } \\ \text { Patrimoine de l'édition }\end{array} \\ \begin{array}{l}\text { 395 Wellington Street } \\ \text { Ottawa ON K1A 0N4 } \\ \text { Canada }\end{array} & \begin{array}{l}\text { 395, rue Wellington } \\ \text { Ottawa ON K1A 0N4 } \\ \text { Canada }\end{array}\end{array}$

Your file Votre référence ISBN: 978-0-494-47509-6

Our file Notre référence

ISBN: 978-0-494-47509-6

NOTICE:

The author has granted a nonexclusive license allowing Library and Archives Canada to reproduce, publish, archive, preserve, conserve, communicate to the public by telecommunication or on the Internet, loan, distribute and sell theses worldwide, for commercial or noncommercial purposes, in microform, paper, electronic and/or any other formats.

The author retains copyright ownership and moral rights in this thesis. Neither the thesis nor substantial extracts from it may be printed or otherwise reproduced without the author's permission.
AVIS:

L'auteur a accordé une licence non exclusive permettant à la Bibliothèque et Archives Canada de reproduire, publier, archiver, sauvegarder, conserver, transmettre au public par télécommunication ou par l'Internet, prêter, distribuer et vendre des thèses partout dans le monde, à des fins commerciales ou autres, sur support microforme, papier, électronique et/ou autres formats.

L'auteur conserve la propriété du droit d'auteur et des droits moraux qui protège cette thèse. $\mathrm{Ni}$ la thèse ni des extraits substantiels de celle-ci ne doivent être imprimés ou autrement reproduits sans son autorisation.
In compliance with the Canadian Privacy Act some supporting forms may have been removed from this thesis.

While these forms may be included in the document page count, their removal does not represent any loss of content from the thesis.
Conformément à la loi canadienne sur la protection de la vie privée, quelques formulaires secondaires ont été enlevés de cette thèse.

Bien que ces formulaires aient inclus dans la pagination, il n'y aura aucun contenu manquant.

\section{Canada}


The undersigned recommend to the Faculty of Graduate Studies and Research acceptance of the thesis

\section{Design and Analysis of a Hierarchical IP Traceback System}

submitted by

Abes Dabir, B.A.Sc.

in partial fulfillment of the requirements for

the degree Master of Applied Science in Electrical Engineering

Chair, Department of Systems and Computer Engineering

Thesis Supervisor

Carleton University

January 15, 2009 


\section{Abstract}

In this thesis, we present the detailed design and analysis of our solution to the IP traceback problem. We adopt and enhance, at the Autonomous System (AS) level, a path signature generation method which was proposed at the router level to primarily provide a means of filtering attack traffic. Our solution assumes a secure BGP routing infrastructure to exchange authenticated messages in order to learn path signatures. This solution is hierarchical in the sense that it works at the AS-level first, then once a small list of possible source ASes is identified, those ASes are queried and traceback is performed within each AS to prune the list down to the actual source. We envision the local adoption of a separate, yet complementary, traditional traceback system at each AS. Using simulation results we demonstrate that our solution is practical since it reduces - as a first step - the search space from the entire router space of the Internet to an AS-list that is only a very small fraction of all possible ASes. We go on to propose a means of using more than 16 bits of the IP fragmentation fields which are traditionally used by various IP traceback systems. We present results based on using various sizes for the marking field, as well as varying number of total marks and different sizes for each mark. 


\section{Acknowledgements}

I would like express my deep gratitude and sincere thanks to my supervisor, Professor Ashraf Matrawy, for his consistent guidance, support, and encouragement during the course of my thesis. Thanks to him I learned the skills necessary to conduct research in a professional manner.

I would also like to thank my family, especially my parents, Hassan and Farideh, for their encouragement, support, and many sacrifices which have allowed me to reach this stage in my life. 
To my parents: Hassan and Farideh 


\section{Contents}

Acceptance Sheet

Abstract

Acknowledgements iv

Table of Contents vi

List of Tables

xi

List of Figures

xiii

List of Acronyms

Xv

1 Introduction

1.1 Motivation . . . . . . . . . . . . . . . . . 1

1.2 Thesis Contributions ....................... 2

1.3 Thesis Outline ....................... 5 
2 Background on IP Traceback Technologies $\quad 7$

2.1 IP Traceback Categories .................. 8

2.1.1 Network Based IP Traceback . . . . . . . . . . . . . 9

2.1.2 Packet Marking Based IP Traceback . . . . . . . . . . . . . 10

2.1.3 Packet Logging Based IP Traceback . . . . . . . . . . . . . . 10

2.2 Probabilistic Packet Marking . . . . . . . . . . . . . . . . . . 10

2.2.1 Original PPM Approach . . . . . . . . . . . . . . 10

2.2.2 Advanced and Authenticated Marking Scheme . . . . . . . . 12

2.2 .3 Fast Internet Traceback . . . . . . . . . . . . . . 12

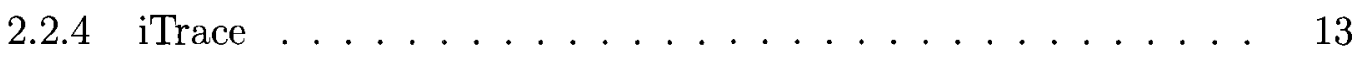

2.2.5 Intention Driven ICMP Traceback . . . . . . . . . . . . . 14

2.3 Deterministic Packet Marking . . . . . . . . . . . . . 15

2.3.1 Original DPM Approach . . . . . . . . . . . 15

2.3.2 Stateless Single-Packet IP Traceback . . . . . . . . . . . . 16

2.3.3 AS-Level IP Traceback Using Bloom Filters . . . . . . . . . . 17

2.4 Packet Logging Based IP Traceback . . . . . . . . . . . . . . . . . 18

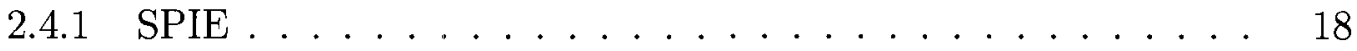

2.4.2 Layer 2 Extension to Hash-Based IP Traceback . . . . . . . 19

2.4.3 AS-Level IP Traceback Using SPIE . . . . . . . . . . . . . 20

2.5 IP Spoofing Mitigation Related Work . . . . . . . . . . . . . . . 21

2.5.1 Ingress and Egress Filtering ... . . . . . . . . . 21 
2.5.2 Unicast Reverse Path Forwarding . . . . . . . . . . . . 22

2.5 .3 TCP Intercept. . . . . . . . . . . . . . . 23

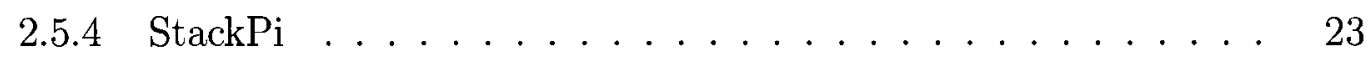

2.5.5 Spoofing Prevention Method . . . . . . . . . . . 23

2.5.6 Route-Based Distributed Packet Filtering . . . . . . . . . 26

2.5.7 Defeating Distributed Denial-of-Service Attack with Deterministic Bit Marking . . . . . . . . . . . . 27

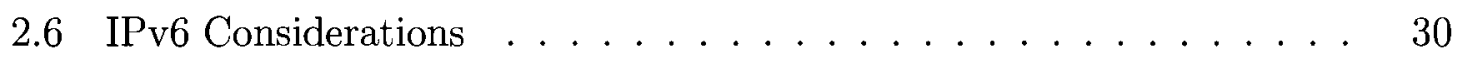

2.7 Discussion on Presented Schemes _. . . . . . . . . . . . . 32

3 Design and Architecture of the Hierarchical IP Traceback System 34

3.1 Overview of Our Approach . . . . . . . . . . . . 34

3.2 Routing Infrastructure Considerations . . . . . . . . . . . 35

3.2.1 Secure BGP Routing Environment . . . . . . . . . 35

3.2.2 Using BGP to Transmit Capability Information . . . . . . 36

3.3 Finding Storage Space Inside of an IP Packet $\ldots \ldots \ldots$

3.4 Marking Packets - Simple Strategy . . . . . . . . . . . . . . 43

3.5 Marking Packets with Support for Incremental Deployment . . . . . . 44

3.6 Further Enhancements to Our Marking Strategy . . . . . . . . . 45

3.7 Associating a Path Signature with its Source AS . . . . . . . . 47

3.7.1 Learning AS-Path Signatures $\ldots \ldots \ldots \ldots \ldots$

3.7.2 Single-Homed and Multi-Homed Customer Considerations . . $\quad 55$ 
3.8 Spoofing Detection and Other Security Considerations . . . . . 58

3.9 Identifying the Origin AS (IP Traceback) . . . . . . . . . . 59

4 Simulation Models and Methodology $\quad 63$

4.1 Topology Generation ................... 63

4.1 Average AS Path Length . . . . . . . . . . . . 66

4.2 NS-2 Modifications . . . . . . . . . . . . . . . . 68

4.2.1 Traffic Agents . . . . . . . . . . . . . . 69

4.2.2 Modifying Packets Enroute . . . . . . . . . . . 69

4.3 Simulation Setup . . . . . . . . . . . . 70

4.3.1 Test Scenarios Executed . . . . . . . . . . . 72

4.3.2 Simulation Phases ................ 74

4.4 Analyzing Simulation Results . . . . . . . . . . . . . 75

5 Results and Performance Analysis with Full Deployment $\quad 77$

5.1 Traceback Using the Simple Marking Scheme . . . . . . . . . . . 78

5.2 Effect of Number of Bits Per AS Mark and Number of Marks . . . . 79

5.3 Effect of Marking Strategy . . . . . . . . . . . . 80

6 Results and Performance Analysis with Partial Deployment $\quad 82$

6.1 Marking Field Length . . . . . . . . . . . . . . 83

6.2 Effect of Topology . . . . . . . . . . . . . 86

6.3 Varying the Number of Marks Recorded . . . . . . . . . . . 88 
6.4 Effect of Marking Field on Learning Path Signatures . . . . . . . . . 92

7 Conclusions and Future Work $\quad 94$

7.1 Conclusions ........................... 94

7.2 Future Research . . . . . . . . . . . . . . . . . . . . 96

$\begin{array}{ll}\text { A NS-2 Modification Summary } & 97\end{array}$

$\begin{array}{ll}\text { Bibliography } & 100\end{array}$ 


\section{List of Tables}

5.1 Traceback results with full deployment (simple marking strategy, 16bit marking field 4 marks $\ldots \ldots \ldots \ldots \ldots$. . . . . . 79

5.2 Effect of increasing the size of each AS mark on the list of potential source ASes (simple marking strategy, 4 marks in total) . . . . . . 80

5.3 Effect of increasing the size of each AS mark on the traceback success rate (simple marking strategy, 4 marks in total) $\ldots \ldots \ldots \ldots$

5.4 Traceback results with full deployment (legacy-supporting marking strategy, 16-bit marking field, 4 marks) … . . . . . . . . 81

6.1 Traceback results with partial deployment (Inet3 topology with 5000 ASes, 16-bit vs 31-bit marking field, 4 marks) $\ldots \ldots \ldots \ldots$

6.2 Comparing traceback results between the Inet3 and Brite topologies (31-bit marking field, 4 marks) . . . . . . . . . . . . . .

6.3 Traceback results with partial deployment (Brite topology with 5000

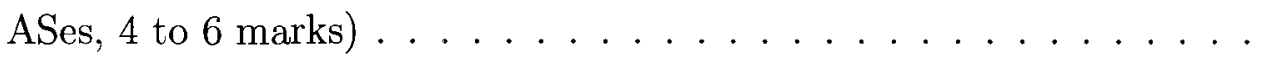


6.4 Traceback results with partial deployment (Inet3 topology with 5000

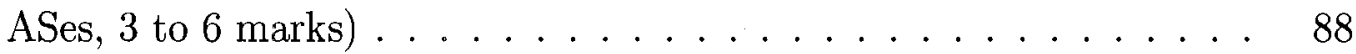

A.1 List of all ns-2 files modified to implement our design . . . . . . . . . 99 


\section{List of Figures}

2.1 Classification of IP traceback schemes . . . . . . . . . . . . . . 8

3.1 Structure of the BGP extended communities path attribute . . . . 38

$3.2 \quad$ IPv4 header $\ldots \ldots \ldots \ldots \ldots \ldots \ldots \ldots \ldots \ldots \ldots$

3.3 Basic marking scheme at the AS-level . . . . . . . . . . . . . . 44

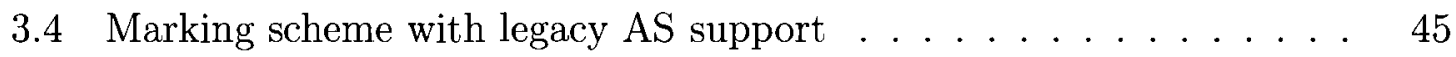

3.5 On-demand request for path signature by AS 5 from origin AS 1 (assuming simple marking strategy) $\ldots \ldots \ldots \ldots \ldots$

3.6 Path signature generation by a supporting AS on behalf of its neighbouring legacy ASes $\ldots \ldots \ldots \ldots \ldots \ldots$

3.7 Examples of customer network connectivity to ISP $\ldots \ldots \ldots 56$

3.8 Path signature matching methods for IP traceback $\ldots \ldots \ldots$

4.1 Histogram of mean distances in the Inet3 topology . . . . . . . . . 65

4.2 Histogram of AS degrees in the Inet3 topology (only showing up to a

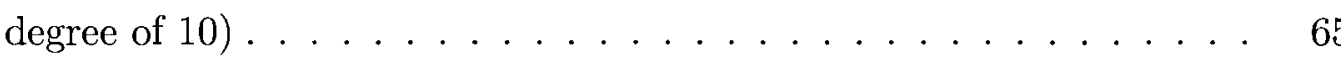


4.3 Histogram of mean distances in the Brite topology . . . . . . . . . 67

4.4 Histogram of AS degrees in the Brite topology (only showing up to a degree of 10$) \ldots \ldots \ldots \ldots \ldots \ldots \ldots$

6.1 Comparison of traceback success rates using AMS and LMS methods (Inet3 topology, 16-bit marking field, 4 marks) . . . . . . . . . . 84

6.2 Comparison of traceback success rates using AMS and LMS methods (Inet3 topology, 31-bit marking field, 4 marks) $\ldots \ldots \ldots \ldots$. . .

6.3 Comparing AMS traceback success rates between Inet3 and Brite topologies $(4$ marks $) \ldots \ldots \ldots \ldots \ldots \ldots \ldots \ldots$

6.4 Comparing LMS traceback success rates between Inet3 and Brite topologies $(4$ marks $) \ldots \ldots \ldots \ldots \ldots \ldots \ldots \ldots \ldots$

6.5 LMS traceback success rates comparison (Brite topology, 4 to 6 marks) 90

6.6 LMS traceback success rates comparison (Inet3 topology, 3 to 6 marks) 91

6.7 Comparison of traceback success rates using AMS and LMS methods (Brite topology, 30-bit marking field, 5 marks) $\ldots \ldots \ldots$. . . . 92 


\section{List of Acronyms}

$\begin{array}{ll}\text { AAM } & \text { Advanced and Authenticated Marking } \\ \text { ACL } & \text { Access Control List } \\ \text { AMS } & \text { All Matching Signatures } \\ \text { AS } & \text { Autonomous System } \\ \text { BGP } & \text { Border Gateway Protocol } \\ \text { DBM } & \text { Distributed Bit Marking } \\ \text { DF } & \text { Don't Fragment flag } \\ \text { DDoS } & \text { Distributed Denial of Service } \\ \text { DoS } & \text { Denial of Service } \\ \text { DPF } & \text { Distributed Packet Filtering } \\ \text { DPM } & \text { Deterministic Packet Marking } \\ \text { FIT } & \text { Fast Internet Traceback } \\ \text { GBF } & \text { Generalized Bloom Filter } \\ \text { HMAC } & \text { Hash Message Authentication Code } \\ \text { IANA } & \text { Internet Assigned Numbers Authority }\end{array}$




\begin{tabular}{|c|c|}
\hline ICMP & Internet Control Message Protocol \\
\hline IDS & Intrusion Detection System \\
\hline IOS & Internetwork Operating System \\
\hline IP & Internet Protocol \\
\hline IRV & Interdomain Route Validation \\
\hline ISP & Internet Service Provider \\
\hline ITU & International Telecommunication Union \\
\hline LMS & Longest Matching Signature \\
\hline MAC & Media Access Control \\
\hline MD5 & Message-Digest algorithm 5 \\
\hline MF & More Fragments flag \\
\hline NAT & Network Address Translation \\
\hline nem & Network Manipulator \\
\hline PKI & Public Key Infrastructure \\
\hline PPM & Probabilistic Packet Marking \\
\hline psBGP & Pretty Secure Border Gateway Protocol \\
\hline $\mathrm{RFC}$ & Request for Comments \\
\hline S-BGP & Secure Border Gateway Protocol \\
\hline so-BGP & Secure Origin Border Gateway Protocol \\
\hline SPIE & Source Path Isolation Engine \\
\hline SPM & Spoofing Prevention Method \\
\hline
\end{tabular}


TCP

TTL

ns-2

UDP uRPF
Transmission Control Protocol

Time To Live

Network Simulator 2

User Datagram Protocol

Unicast Reverse Path Forwarding

xvii 


\section{Chapter 1}

\section{Introduction}

\subsection{Motivation}

As is evident from studies and news coverage over the past decade or so, there is a considerable level of malicious/criminal activity (e-crimes) that take place over the Internet, such as DoS, DDoS, worm and virus epidemics, extortion, espionage, etc. The cost of such e-crimes can reach many millions, if not billions of dollars. The number of such incidents is on the rise and this puts ever more pressure on law enforcement and potential victims to counter or defend themselves against such e-crimes.

Different categories of attack require different types of defenses and response mechanisms. IP traceback, as defined by Savage et al. in [49], is a line of research that tries to aid network operators when they are subjected to attack traffic whose IP 
source addresses are spoofed. IP traceback allows network operators to carry out forensics on already received, or incoming traffic in order to identify the true source of the traffic, as well as potentially identifying the network path the packet(s) has taken. Typically the main avenues of attack that use spoofed source addresses are DoS and DDoS attacks. DoS and DDoS attacks are a major area of concern for many network operators. Surveys carried out by Arbor Networks in [36] indicate that from August 2007 through July 2008, ISPs spent most of their available security resources combating DDoS attacks.

While many solutions have been proposed over the years, they all suffer from certain shortcomings and other issues that impact their feasibility. These shortcomings, such as lack of legacy support, poor support for incremental deployment, high processing overhead or storage requirements, are discussed in more detail in Chapter 2 when we discuss a large number of currently proposed solutions. We believe there is room for improvement in this area, and hence present our own solution to the IP traceback and spoofing detection problems with the goal of concurrently addressing many of these shortcomings.

\subsection{Thesis Contributions}

In this thesis we present our solution to the IP traceback problem. Our approach is a hierarchical one that uses deterministic packet marking at the Autonomous System (AS) level to identify the AS-path an incoming packet has taken. As well, we can 
narrow down the potential source ASes of the packet to a very small fraction of all potential ASes. At the individual ASes we envision the local adoption of a separate IP traceback system responsible for carrying out traceback within that AS, which would work cooperatively with our approach to prune the list of potential source ASes of an attack packet. Unlike many other approaches, ours supports incremental deployment. Using simulation results we show that it performs well even in a partial deployment scenario.

In StackPi [59] Perrig et al. proposed a method to create a unique router-path signature for the path taken by a packet. They used this signature to filter out attack packets having taken the same path and detect spoofed IP source addresses; they also suggested it can be used for traceback. We explore the idea of creating a unique ASpath signature by adopting, and building upon their technique. We believe doing this at the AS level makes implementation much more practical. One significant advantage of moving from the router level approach to the AS level is that AS-paths are highly stable [20]. This means the path signatures we learn do not change nearly as often as they would at the router level. Another advantage is that a packet traverses far fewer ASes than individual routers, therefore each AS can use more bits to place its mark in a packet than the number of bits each router could use for its mark. This would in turn make for more distinct markings.

While StackPi primarily focused on providing a means of filtering DDoS attacks, which also applies to our technique, we propose a more practical and detailed means 
of learning which path signatures belong to which sources in order to detect IP spoofing. We primarily explore the possibility of using such a technique to carry out IP traceback on suspect packets. As well, we go on to suggest a method which would allow using more bits from the 32-bit fragmentation fields for marking. We present simulation results evaluating the impact of choosing variables such as the number of bits used per AS mark and the total number of AS marks placed in each packet on the accuracy of the results.

Overall, our contributions can be summarized as such:

- An AS-level path signature generation technique that benefits from BGP path stability

- Proposed two detailed methods for learning AS-path signature in order to detect packet spoofing and perform IP traceback

- Integrating research in the BGP routing security field with the StackPi proposal to facilitate its functionality and better support incremental deployment

- Proposed a means to use more of the IP fragmentation bits for marking compared to existing proposals

- Proposed two techniques for AS-path signature comparison when performing IP traceback

- Simulation-based evaluation of IP traceback using AS-path signatures

- Analysis of the impact of variables such as the number of bits allocated to each mark, and the total number of recorded marks on the accuracy of the results 
The majority of solutions currently proposed do not offer this integrated solution set, hence we believe ours to be a good candidate for future adoption, or serve as the basis for future work in this area. We have submitted a conference paper [22] based on the research presented in this thesis, which has been accepted in IEEE ICC 2009. We have also produced a journal paper [23] which is under review.

\subsection{Thesis Outline}

The rest of this thesis is organized as follows:

In Chapter 2 we present some of the prominent related work in this area. These works include both IP traceback and spoofing mitigation techniques. We go on to discuss IPv6 considerations for this line of work.

In Chapter 3 we present the design details of our hierarchical IP traceback solution. We discuss some of the requirements for our approach as it relates to the BGP routing infrastructure. We explain what parts of the IP header we use to store traceback marks, as well as how to use more portions of the IP header than what is commonly used by various IP traceback approaches. We present our packet marking technique, and propose two methods for tracing back the marked packets back to their source AS.

In Chapter 4 we discuss how we generated our topologies, and the modifications made to the tool used for running the simulations. We go into the details of the test cases used, and the setup of each simulation. 
Chapter 5 contains the results of our testing where we have full deployment of our solution by all ASes. In Chapter 6 we present similar simulation results for partial deployment scenarios. In these chapters we present the effects of varying variables such as the number of marks, the length of the marking field, changing the marking strategy, changing the topology, etc.

Finally we present our concluding remarks in Chapter 7, along with possible directions for future research. 


\section{Chapter 2}

\section{Background on IP Traceback}

\section{Technologies}

In this section we provide an overview of a number of approaches proposed in the area of IP traceback over the years. We first identify the different categories of solutions, and then discuss a few examples of prominent works in each category.

We will also discuss a number of works which are geared towards combating IP spoofing only and do not provide an IP traceback mechanism. Since our approach touches on both IP traceback as well as spoofing, it is important to discuss a number of works in this field as well.

Several detailed survey papers have been published regarding the prominent IP traceback approaches [14][30][27]. In this section we will put more emphasis towards discussing approaches that are more relevant to our work. 
The overwhelming majority of the work proposed thus far, and discussed here, is based solely on IPv4. In the final part of this section we will discuss some of the IP traceback proposals with IPv6 in mind. We will touch upon the differences between the IPv4 and IPv6 protocols as they relate to the categories of traceback schemes discussed.

\subsection{IP Traceback Categories}

Figure 2.1 shows the main categories of IP traceback schemes in squares, and the most prominent proposal in each category is displayed in a circle. We will discuss these in the following sections.

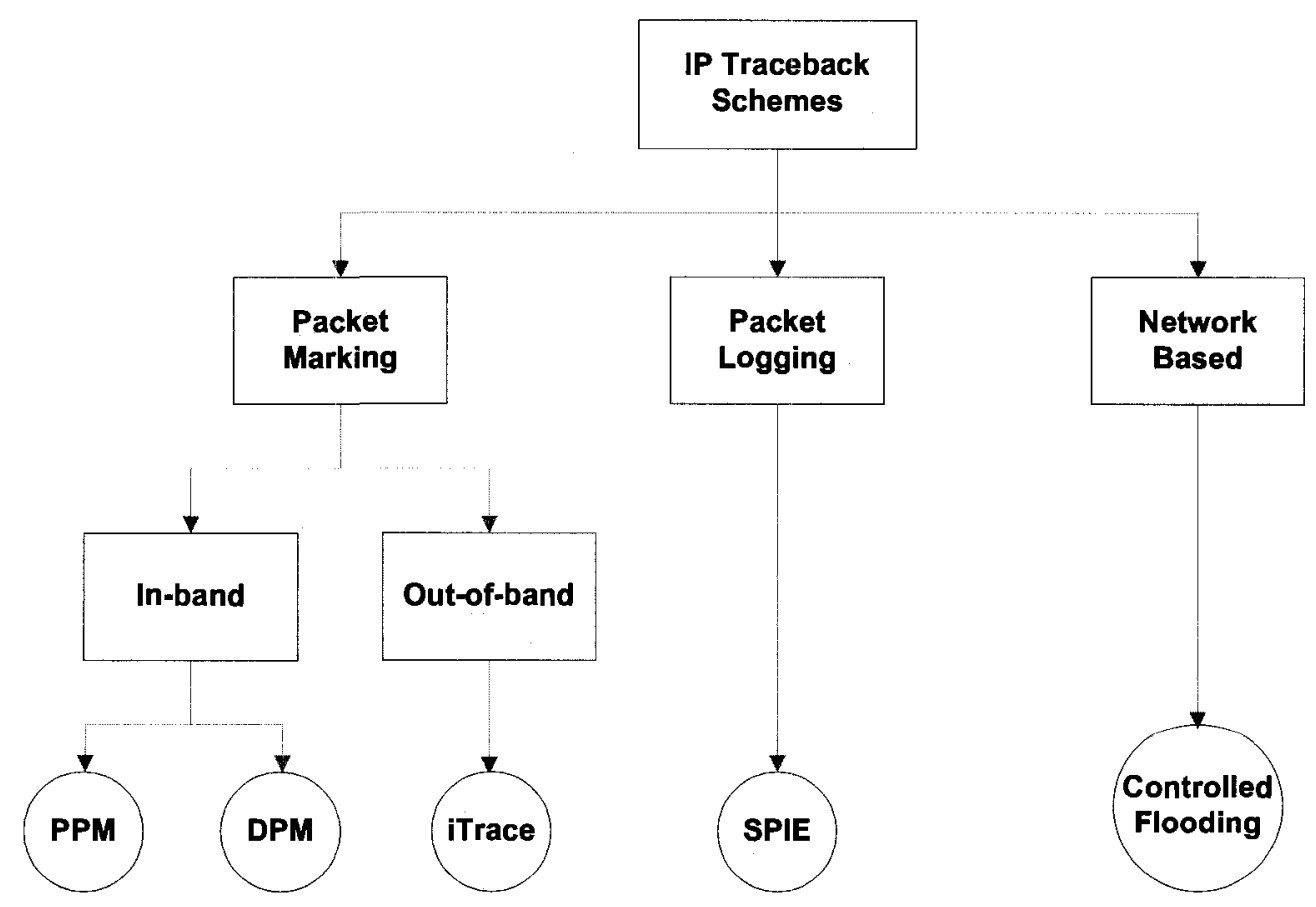

Figure 2.1: Classification of IP traceback schemes 


\subsubsection{Network Based IP Traceback}

Network based IP traceback mechanisms rely on the network structure itself, and already available features in networking equipment to perform traceback. This category of IP traceback involves rather crude, manual approaches, and is not the subject of new research in this field.

Controlled Flooding, presented in [19], is a classic network based traceback approach. It relies on the assumption that the incoming links to the router closest to the victim are heavily loaded. A large burst of traffic is applied to each of these incoming router links in successive order. If after applying the traffic burst to an incoming link of the router we observe a decrease in the rate of attack packets arriving at the victim, then this means the link we are testing carries attack traffic. We would move on to the upstream router the link we have identified is attached to and repeat the same procedure to discover which of that router's incoming links is carrying attack traffic. Applying this procedure recursively should eventually lead us to the network edge router where attack traffic first enters the network. While potentially effective against DoS attacks, this is a very manual and time consuming procedure. It will not work against DDoS attacks which would involve multiple links of the same router carrying attack traffic. On the plus side, this procedure requires no modifications to the individual packets, or any storage in the network. 


\subsubsection{Packet Marking Based IP Traceback}

All IP traceback schemes other than the network based variety rely on storing certain information about each packet, or flow of packets, in order to carry out traceback. Packet marking approaches generally incur a small amount of overhead at the routers in exchange for large processing overhead at the final victim which has to use all the marked information in order to reconstruct the attack path. In the in-band packet marking schemes this information is stored in the header of each individual packet before it is forwarded, while in the out-of-band marking schemes the information is put into a separate new packet and sent to the victim.

\subsubsection{Packet Logging Based IP Traceback}

In contrast to the packet marking IP traceback schemes, logging based approaches place the storage requirement on intermediate nodes along the path. In these approaches, either the entire packet is logged, or more commonly, packet fingerprints are stored in a specialized data structure and later used for traceback.

\subsection{Probabilistic Packet Marking}

\subsubsection{Original PPM Approach}

In [49] Savage et al. introduce the original Probabilistic Packet Marking (PPM) scheme. In this scheme, at each router along the path of a packet, there is a probability 
$p$ that this router will mark the packet with partial information about the network edge it is being forwarded on. This edge information consists of the IP address of the current router, the IP address of the next router along the path, and a hop count to the victim which will be incremented by every supporting router along the path. The mark is placed in the 16-bit identification field of an IPv4 packet. While this does break IP fragmentation, this choice is based on measurements suggesting that less than $0.25 \%$ of IP traffic is fragmented. The very small size of this field compared to the edge information that needs to be recorded in it limits how much information can be stored when marking, hence only partial information can be accommodated in the packet. Savage et al. propose a clever means to fragment a single piece of edge information across multiple packets, and have these fragments reconstructed at the victim. However, therein lies a lot of the weaknesses of their approach.

The victim is expected to collect all the marks it receives in incoming packets. Due to the probabilistic nature of the marking, only a fraction of the packets will contain a mark. Using the collected marks, the victim will execute a reconstruction algorithm to reconstruct the path of the attack packets. Reconstruction is a very time consuming process, requires a large number of packets (somewhere in the order of several thousand), results in a large number of false positives, and does not work well when the number of attack sources is high. Nevertheless, this work serves as a foundation for much of the follow up work in the field of IP traceback. 


\subsubsection{Advanced and Authenticated Marking Scheme}

This work was significantly improved by Song and Perrig in [51]. Their Advanced and Authenticated Marking (AAM) scheme assumes the victim knows the map of its upstream routers. Based on this assumption they can enhance the encoding principles put forward by Savage et al. and make them efficient and accurate enough to scale up to thousands of simultaneous attackers. This scheme requires a few thousand packets as compared to the original PPM approach by Savage et al. which requires several thousand packets to initiate traceback. As well, the computation time has been reduced to about 100 seconds compared to the original approach which took a few days to compute the attack route. Incremental deployment however is more difficult with this approach than the original PPM approach [58].

\subsubsection{Fast Internet Traceback}

Fast Internet Traceback (FIT) [58] is yet another probabilistic packet marking scheme that tries to improve on previous schemes in terms of deployability and performance. Compared to the previous two PPM mechanisms mentioned, FIT requires tens of packets to perform traceback, has better legacy support, and scales up to thousands of attackers. It achieves these improvements over the previous PPM schemes by manipulating the IP header's TTL field. It does overwrite the TTL field, however, based on their analysis this doesn't cause major problems. Although, in some cases packets with certain default TTLs, may persist in routing loops longer than they 
would have without FIT in effect. Also, because of the requirement to overwrite the TTL field, tools such as traceroute which are very sensitive to the TTL values and use that as the basis for their functioning will not work. These would require new implementations to work with FIT.

\subsection{4 iTrace}

One notable out-of-band packet IP traceback scheme is called iTrace [16]. Every router statistically picks a packet it is forwarding (they recommend 1 in every 20,000), and generates a new ICMP message destined to the same destination as the packet. This ICMP message would contain as much of the traced packet as possible, a timestamp, authentication information, as well as information on the previous and next hops. The victim would reconstruct the attack path based on the ICMP messages received. The previous and next hop information in the iTrace packets received by the victim would be pieced together across a chain of iTrace packets in order to identify the attack route. The authentication options suggested, increasing in security strength from left to right, are: cleartext random strings, HMAC, digital signatures backed by a PKI.

This scheme does not work against denial of service attacks that use very few packets. It is best suited to a situation where a relatively small number of sources are generating a significant amount of traffic. It remains a possibility that the one packet chosen among 20,000 packets at a router to generate an iTrace packet based on, is 
not actually an attack packet. Ideally iTrace packets should be generated based on attack packets.

\subsubsection{Intention Driven ICMP Traceback}

In [39] Mankin et al. suggest a number of improvements over the iTrace method. They focus on improving the usefulness of the iTrace packets generated. Their first improvement involves sending iTrace packets only to destinations that the current router knows support iTrace and are interested in receiving these packets. They propose the use of the BGP protocol to distribute knowledge about which routers want to receive iTrace packets.

In the original iTrace, once a packet statistically causes an iTrace packet to be generated at a router, this iTrace packet would be sent to the triggering packet's destination address. In this new scheme, the generated iTrace packet's destination is decoupled from the destination IP of the triggering packet. A router maintains a list of routing entries that want to receive iTrace packets. When a packet statistically triggers the generation of an iTrace packet (the iTrace packet isn't generated right away), the router randomly selects one of these routing entries to be the next to receive an iTrace packet. The choice of which routing entry should get the next iTrace packet has nothing to do with the contents of the packet that statistically triggered this selection. The very next normal packet forwarded by this router to the same network as the selected routing entry will cause a corresponding iTrace packet 
to be generated and sent to this packet's destination IP address, with as much of this packet copied into it as possible. This improvement causes more potential victims to receive iTrace packets as opposed to a few victims receiving duplicate packets while others receive none.

When selecting the next routing entry to receive an iTrace packet, this scheme applies a level of bias towards destinations with longer AS paths. They argue that there is relatively little value in generating iTrace packets for destinations that are 3 hops or less from the generating router.

\subsection{Deterministic Packet Marking}

\subsubsection{Original DPM Approach}

Deterministic Packet Marking (DPM) is another variation of this category where a router marks every packet it forwards rather than only some packets with a given probability. In [15], Belenky et al. proposed a DPM approach where only ingress routers (border routers handling incoming traffic from outside the network) would mark packets rather than all routers along the path. The mark would contain partial identification information about the incoming interface on the ingress router. Every time the packet enters a new network, its mark would be overwritten by that network's ingress router. Using this system a victim would be able to use the collected marks to deduce the address of the ingress router in the victim's own network where the attack 
traffic is coming through. The researchers estimate this scheme requires roughly 55 marked packets for the victim to identify each ingress router interface attack traffic is coming from. This would allow the victim to apply the proper filters at the entry point of the attack traffic to its network. This approach does not aim to trace attack traffic all the way back to the source network.

\subsubsection{Stateless Single-Packet IP Traceback}

In [37] Laufer et al. present an interesting deterministic packet marking approach with the goal of being able to trace a single packet to its source. Their approach involves embedding a new data structure, which is based on the well known Bloom filter [17], into the IP header. They call this new data structure a Generalized Bloom Filter (GBF). At every hop, the router places the hash of its IP address in the GBF contained in the packet header. Path reconstruction is done incrementally at every distance level, starting with the victim's direct neighbours. Whichever of the victim's direct neighbours has the hash of its IP address present in the GBF will have to check with its own neighbours to see which of them appear in the GBF. Recursively following this process should lead to the source of the packet. The biggest hurdle to this approach is the issue of where to place the GBF. The authors are not completely clear on the size of the GBF, or their choice as to where to place it in the IP header. However, we surmise that the GBF is far larger than the 16-bit IP identification field and would have to accommodated as an IP option. This is not a feasible choice 
however due to slow processing of IP options, especially since this would have to be done at every hop.

\subsubsection{AS-Level IP Traceback Using Bloom Filters}

The above proposals all work at the router level. One of the in-band packet marking proposals that works at the AS-level, which is where our own proposal works, is [21]. This proposal builds upon the work done by Laufer et al. in [37], which was discussed in Section 2.3.2. It shares the same weakness as that proposal when it comes to placing the relatively large GBF in the packet. In this approach each packet carries GBF in its header, and every AS through which the packet passes places its mark in it. At the destination AS, the victim can check which of its neighboring ASes have their mark present in the bloom filter. Then the victim would check to see which of the identified AS' neighboring ASes have their mark in the packet, and so on until it reaches the source AS. In terms of impediments to implementation, similar to the work this is based off, once again there is the problem of where to place the GBP in the IP header. The main problem is still the fact that the GBF added to each packet in order to store the marks is relatively large compared to the size of many small packets frequently transmitted, as well as the fact that it would have to be added as an IP option which significantly slows down router performance. 


\subsection{Packet Logging Based IP Traceback}

\subsubsection{SPIE}

The underlying packet logging technique that forms the basis for other related works was carried out by Snoeren et al. in [50] and is called SPIE. The revolutionary idea suggested by Snoeren et al. was to store fingerprints of each packet in a special data structure called a Bloom filter on each router that the packet traverses. Storing packet fingerprints in Bloom filters significantly cuts down on the storage requirements for packet logging at the cost of more processing overhead and chance of false positives. Their calculations suggested that the required amount of storage for a router link would be around $0.5 \%$ of that link's capacity. Even at such levels, when dealing with high speed links traffic can only be stored for a very short period of time.

The main disadvantages of this approach are the large amount of space required for storing the Bloom filters, and the added processing for each packet which may require specialized hardware. Legacy support is also an issue with SPIE. Consider an example where we need to find out which of our 10 neighbouring routers forwarded a packet. If 4 of these routers are legacy, then we would have to poll our 6 SPIE supporting direct neighbours, as well as all the neighbouring routers of the 4 legacy routers, which may themselves be legacy. This can result in having to contact a large number of routers at each stage.

Some of the advantages of this approach include the ability to trace even a single 
packet, moving the processing overhead from the victim to the routers, and leaving the packets unmodified.

\subsubsection{Layer 2 Extension to Hash-Based IP Traceback}

In [32] Hazeyama et al. present an extension to hash-based IP traceback systems, specifically the SPIE system already discussed, aimed at continuing the traceback process at layer 2 to identify the specific node sending attack traffic. IP traceback systems working at layer 3 are at best only capable of identifying the router closest to the attacking node and not the individual node itself.

In this scheme, a leaf router would need to store and maintain a bloom filter to hold layer 2 information, in addition to the existing Bloom filter from SPIE for layer 3 traceback. It would also require two conversion tables for MAC address to network interface mappings on the leaf router, and MAC address to port identifier mappings on the attached layer 2 switches. A leaf router would need to obtain the forwarding databases of the switches attached to it so that it can map port identifiers to MAC addresses on them. It can accomplish this if the switches in question are running Bridge-MIB [24]. When a packet arrives at the leaf router, its signature is computed as in SPIE and stored in the layer 3 bloom filter. The same computed signature is concatenated with information identifying the originating switch port ID and subnet, and is then stored in the layer 2 Bloom filter.

While this scheme adds layer 2 traceability, it increases the already high memory 
and processing requirements of SPIE on a router implementing it. As well, the implementation of layer 2 traceback on a suspect packet is quite a bit more processor intensive than layer 3 traceback with plain SPIE.

\subsubsection{AS-Level IP Traceback Using SPIE}

Another AS-level proposal which uses packet logging is introduced in [28]. In this approach, we have a partial deployment scenario where a certain number of ASes have SPIE deployed. In order to perform traceback on a packet, the victim AS asks it neighbouring ASes to check their own packet logs and tell it which of them sent it the packet. When one of its neighbours replies positively, it would then repeat the same process with that AS' neighbours. If the reconstruction comes across a set of ASes that don't have SPIE deployed and hence can't determine which of them sent it the packet, the victim will have to use its knowledge of the AS topology and ask the SPIE supporting neighbouring ASes of every legacy AS it is currently stuck at, hoping one of them will respond positively. Amongst the disadvantages of this approach are that its success rate drops quickly as the percentage of ASes deploying it goes down, it generates a rather large number of query messages to perform traceback, and that this has to be done for every single packet being traced. 
Background on IP Traceback Technologies

\subsection{IP Spoofing Mitigation Related Work}

\subsubsection{Ingress and Egress Filtering}

Ingress and Egress filtering [34] are the two most common techniques currently used to combat IP source address spoofing. Ingress filtering implemented at an ISP is done from the customer edge site to the ISP. In this approach any customer traffic that has a source address other than what has been assigned to the customer is dropped. This does not prevent spoofed addresses within the customer IP prefix. In some cases ingress filtering may break some services offered by an ISP such as Mobile IP [26].

Egress filtering implemented by an ISP is done from the ISP to the customer edge site. Any traffic going to the customer with source addresses that are assigned to the customer network are dropped.

Both these approaches are based on good-will and good net-citizenship by other networks. They do not provide any assistance to a victim network under attack from traffic passing through networks not implementing these features. As well, since these two techniques are meant to help other networks, this limits the incentives for individual networks to commit to the costs and administrative overhead of enabling them when they may not receive any direct benefit themselves. The effectiveness of ingress and egress filtering depends on large scale adoption by networks on the Internet. Unless ingress filtering is carried out by almost all networks on the Internet, it is not an effective DDoS prevention strategy [42]. 
If the victim network implements this type of filtering at its own edge, it can filter out incoming attack packets that have source addresses belonging to its own network. However, this is only a very small fraction of all possible spoofed source addresses. It will not be able to filter out packets with spoofed source addresses belonging to other networks.

\subsubsection{Unicast Reverse Path Forwarding}

Unicast Reverse Path Forwarding (Unicast RPF) [10] is a router feature for mitigating malformed and spoofed IP packets. uRPF can be seen as an extension of ingress and egress filtering strategies. When this feature is enabled on a router, upon receiving a packet the router checks the source IP address and verifies that the source IP network is reachable through the same interface (must be best return path) on which the packet arrived. If this is not the case then the packet is dropped.

Among the limitations of $\mathrm{uRPF}$ are that it is best implemented in the presence of symmetric paths. Asymmetric paths require ACLs to prevent packets from being dropped and complicate its implementation. Furthermore, simply ensuring that the incoming interface is the best return path to the packet's claimed source network does not guarantee that the source IP is not spoofed. 


\subsubsection{TCP Intercept}

TCP Intercept [9] is a feature available in the Cisco IOS software. This feature is meant to protect servers running TCP services from TCP SYN-Flood type DDoS attacks. It does this by intercepting and validating TCP connection requests. If a TCP connection can be fully established, it indicates that the source IP address is not spoofed. Two limitations of this approach are that it puts a heavy burden on the router that has it enabled, and that it only works for TCP type traffic.

\subsubsection{StackPi}

Another relevant security proposal we would like to discuss is StackPi [59]. This work is primarily intended to create a unique path signature for the router-path taken by a packet, and use this signature to filter out attack packets having taken the same path. It does this by deterministically marking the IP identification field of a packet at every router that forwards it. Each router shifts the current bits in the IP identification field left by $n$ bits before putting its own $n$-bit mark at the rightmost end of this field. While this work leaves the door open to IP traceback, it does not explore this field itself and focuses on filtering.

\subsubsection{Spoofing Prevention Method}

The Spoofing Prevention Method (SPM) [18] is solely an anti-spoofing method, meaning it does not support traceback. In SPM, each source and destination AS pair are 
required to share a key, which is chosen and placed in the packet (they call it tagging) by the source AS and is verified at destination AS. This key is a simple string of 32 , or 16 bits. Verifying the key at the destination AS simply involves checking to see if the packet contains the same key as what is expected of the AS it is claiming to have originated from. There is no cryptography involved in this verification process.

The researchers suggest placing this key in the packet header as either a 32-bit value in the IP options field, or as a 16-bit value in the IP fragmentation ID field. Placing the key in the IP options field is not feasible due to the significant overhead it would introduce, therefore the latter approach is the only feasible choice. This would limit the key length to 16 bits.

In SPM only the source AS tags packets originating from it. It is recommended that the intermediate ASes along the way authenticate the tagged key in packets they forward so as to drop spoofed packets as early as possible in the forwarding process. This however means that an intermediate AS would need extensive knowledge about the keys used between each source and destination AS, which could number in the billions and require gigabytes to store, depending on how many ASes adopt SPM. It should also be noted that given a 16-bit key length, and requiring a key for every source and destination AS pair results in a significant number of such pairs sharing the same keys.

Each AS independently chooses the set of keys it will use to mark traffic originating from it. These keys are then communicated with other ASes having implemented 
SPM. They propose two methods for key distribution. In the first method an AS learns which keys to expect from source ASes by monitoring non-spoofed incoming traffic. Incoming traffic is judged to be non-spoofed if it completes the three-way TCP handshake. The second key distribution mechanism uses an active distribution protocol which borrows from architectures proposed by researchers to secure the BGP routing infrastructure. Specifically they base their protocol on the IRV [29] BGP security architecture in which each participating AS has a dedicated central server used to ensure the integrity of BGP information. We discuss the IRV architecture in more detail in Sec. 3.2.1. In SPM such a central server is used to announce keys to other ASes, and receive key announcements from other ASes.

The researchers do not discuss legacy support in detail. Since tagging packets with a key is only done at the source AS, SPM will not have any support for legacy source ASes. It is not possible for intermediate ASes to tag packets having been originated in a legacy AS, because the destination AS looks specifically for a tag corresponding to the source IP address' AS and itself, not some intermediate AS. The address of an intermediate AS which might compensate for a legacy AS by tagging the packet for the first time would not be in the packet itself, therefore the destination AS will not be able to verify the key.

As previously mentioned, this technique does not support IP traceback, therefore a victim AS will not be able to locate, or narrow down the potential sources of spoofed traffic. 


\subsubsection{Route-Based Distributed Packet Filtering}

Route-based distributed packet filtering (DPF) [42] is a novel approach presented to combat both spoofing and allow for IP traceback at the AS level. DPF achieves these goals by using very detailed BGP AS topology and routing information to determine if an incoming packet is valid with respect to its source and destination addresses, given the reachability constraints imposed on it by the aforementioned pieces of information. The approach presented is able to achieve relatively accurate results in the presence of a large number of legacy ASes. This approach, similar to ingress filtering, has issues regarding incentive for individual ASes to implement it. However, unlike ingress filtering DPF can deliver much better results given roughly $20 \%$ deployment.

Using the same detailed BGP AS topology and routing information constraints, DPF can carry out IP traceback at the AS level on spoofed traffic. When comparing their results with ours under similar circumstances, our approach presented in this thesis can deliver traceback results which are quite a bit more accurate. We refer to similar circumstances here because a lot of the results presented in [42] are based on making assumptions that would work in their favor. An example of one such assumption would be that there is only one (shortest path), or very few possible routes from a source AS to a destination AS being used out of all possible non-looped routes.

The main issue with DPF however is gathering the very detailed topology and 
routing constraint information it needs in order to operate. DPF requires source reachability information to function as opposed to just destination reachability information which is what is carried in BGP messages. DPF would require a new protocol to carry such information. As noted by the authors they do not have an answer to the efficient implementability of their solution for IP internets. Nevertheless, DPF presents a number of interesting ideas which can benefit our own solution to narrow down the space of possible source ASes we identify. Similarly, DPF can benefit from our approach as well.

\subsubsection{Defeating Distributed Denial-of-Service Attack with De- terministic Bit Marking}

In [35] Kim et al. propose a router-level deterministic marking strategy that shares a number of similarities with the already discussed StackPi approach. Their approach, which is called deterministic bit marking (DBM), is not meant to detect spoofing, rather it is meant to serve as a means of isolating and discarding DDoS traffic based on the common path signature in these packets. DBM also allows the possibility of IP traceback.

Like StackPi, DBM is also centered around the idea of creating a unique path signature at the router-level for the path a packet travels. Their marking strategy is very different than StackPi's however, and we believe it is inferior as will be explained shortly. Like many other approaches they propose to use the 16-bit IP identification 
field for this mark, although they also present a more advanced version of their approach where they also make use of an additional 13 bits in the IP Fragment offset field.

In DBM, a router randomly chooses $n$ bit positions in the marking field and simply performs an exclusive-OR operation on the value of those bit positions with 1 . In their simulations they used 3 bits, hence we will use the same value here when explaining their technique. The 3 bit positions chosen can be anywhere in the marking field. Once a router selects these 3 bit positions, it will continue to use them for an extended period of time. The very first ingress router is expected to set the entire marking field to 0 before performing its marking. Each router along the path that supports DBM will mark its own randomly selected 3 bit positions, and thus result in a unique path signature at the destination.

IP Traceback can be performed at the victim in DBM by taking a map of all upstream routers, and having knowledge of which bit positions each chose, try to take the received path signature and bit mark it in reverse along each potential path until the marking field becomes all 0s. Among the challenges with this approach are the construction of a map of all upstream routers, and somehow obtaining knowledge of what bit positions they had randomly chosen to mark. There is of course the possibility that multiple paths will result in the marking field becoming all 0s due to signature collisions. It is unclear how processing intensive this reconstruction would be. 
Both StackPi and DBM share the problem of working at the router-level where routing paths are not all that stable and may change often. DBM's reliance on the very first router to set the marking field to all 0s is a major problem which sets it apart from StackPi. DBM's functionality would be significantly reduced in the presence of legacy routers, while StackPi would be much more functional in the same situation. If the very first ingress router in the DBM approach does not set the IP identification field to all $0 \mathrm{~s}$, then there is a good chance some of the randomly initiated values in that field will not be touched by the DBM supporting routers and make it to the final destination. This would cause the path signature in each packet to be completely different. Since the bit positions in DBM can be chosen anywhere in the marking field, it would be very difficult to separate the random bits from the legitimate marking bits. In StackPi since the random initialized bits are constants shifted out of the packet to one side as new marks are placed, it is much easier to deduce the random bits from the legitimate marking bits.

The authors also present a more advanced version of their approach that uses a 29-bit marking field. The extra 13 bits are used for a checksum on the value of the first 16 bits of the marking field plus the value of the TTL field at the time the mark was placed in the packet. The first 16 bits are marked as before. The purpose of this is that if at a DBM supporting router, the DBM checksum in an incoming packet matches the mark, then the packet must have been forwarded from a DBM supporting router which marked it. Otherwise, the packet is considered to be unmarked. The 
problem here is that if there is a single legacy router along the path, as soon as this legacy router decrements the TTL it will invalidate the checksum and the entire packet will be considered unmarked by all subsequent DBM supporting routers.

\subsection{IPv6 Considerations}

While there are clearly differences between IPv4 [43] and IPv6 [25], as far as IP traceback is concerned, much of the same ideas that were applied in the realm of IPv4 also apply in IPv6. There are some modifications that need to be made to these solutions of course.

In [53] Strayer et al. present an IPv6 compatible version of SPIE, the prominent packet logging solution based on Bloom filters. The main difference with the IPv4 version lies in which parts of a packet are used in computing a hash signature to store in the Bloom filter. One interesting observation they make is that the IPv6 packet header does not exhibit as much entropy as the IPv4 header. One reason for this is that the IPv4 identification field, which is set to a random value before transmission by the sending node, is no longer present in the IPv6 header. Consequently in an IPv6 environment SPIE would have to use quite a bit more data from the packet as input into its hash calculation function.

The packet marking approaches are perhaps more affected by moving to IPv6 as opposed to logging-based approaches. Most packet marking approaches in IPv6 attempt to find a header field comparable to the IPv4 identification field. The original 
probabilistic packet marking approach proposed by Savage et al. for IPv4 mentions the 20-bit IPv6 Flow Label field as a viable option to replace the IPv4 identification field when it comes to marking. In [12] for example, the researchers attempt to implement the advanced packet marking approach, which was discussed earlier, in IPv6. IPv6 packet marking approaches that do not use this field, such as [13], instead use the hop-by-hop IPv6 extension header to store the packet mark, which is equivalent to IPv4 options. This is not feasible for the same reasons IPv4 options were not feasible, which is due to the fact that they have to be handled in software [8] and significantly slow down packet processing.

The IPv6 Flow Label field, the specifications of which are extensively discussed in [44], may be used by a source to request special handling by IPv6 routers for a sequence of packets it has labeled. At the IPv6 level, a flow is identified by a 3tuple consisting of a flow label, as well as source and destination addresses. This field however is still experimental and subject to change as the requirements for flow support in the Internet become more clear. If a packet's Flow Label field is used for IP traceback, its contents would only undergo unwanted modification if it passes through a legacy IPv6 router, with flow specific treatment enabled, if the packet's flow label matches an existing one on that router with the same source and destination address pair. It is not clear how probable this might be. Otherwise, using this field for IP traceback in IPv6 should be fine.

While the IPv6 Flow Label field does grant an additional 4 bits compared to the 
16-bit IPv4 identification field, we should keep in mind that IPv6 addresses are 128 bits long as opposed to 32-bit IPv4 addresses. This makes the job of packet marking approaches more difficult when fragmenting edge, or similar, information and placing them across multiple fragments for later reassembly. This causes more false positives, more packets required for IP traceback, and lesser accuracy compared to IPv4.

\subsection{Discussion on Presented Schemes}

In this Chapter we have presented, and evaluated, various IP traceback and spoofing detection schemes. Within this context, we will now briefly discuss the advantages of our solution, which we present in Chapter 3, in order to distinguish it from the rest.

The probabilistic packet marking set of IP traceback solutions we discussed are primarily concerned with reconstructing attack paths based on information gathered from a large number of attack packets. If we select an individual attack packet, these approaches cannot identify which path it came from since an individual packet does not contain enough information to reveal this. Nor can they tell us if an incoming packet is spoofed or not.

In our solution, we aim to allow for per-packet IP traceback. Each packet will contain enough marking information so that we can narrow down its possible source to a small set of ASes. Per-packet IP traceback capability is a highly desired feature in this area. As well, the mapping from marking information to the set of potential source ASes can be done almost instantaneously, which allows for a fast response time. 
PPM-like approaches on the other hand can require a very long time to construct the possible attack paths. The original DPM approach is intended to identify the ingress router of the victim network only, not the actual source. Approaches such as SPIE, which can trace individual messages, require considerable communication between upstream routers to trace each packet.

IP traceback solutions in general do not provide any means to quickly determine if a packet arriving at the victim (or victim network) is spoofed or not. Alternatively, solutions aimed at identifying incoming spoofed packets do not necessarily allow for IP traceback. In our solution we provide both functions.

Scalability and legacy support are of course two important requirements for an effective solution in this area, and we have designed ours to meet these requirements. Scalability is applicable both in terms of deployment, and the ability to trace a large number of attackers under a wide-spread DDoS attack for example.

Our solution of course requires a number of trade-offs to achieve these qualities, however we believe all of these to be realistic and feasible. Our requirements in the areas of additional network bandwidth, processing and storage overhead at routers, and management overhead are all low to moderate when compared to the other works proposed. 


\section{Chapter 3}

\section{Design and Architecture of the}

\section{Hierarchical IP Traceback System}

\subsection{Overview of Our Approach}

The underlying idea behind the marking strategy in our solution is that as a packet traverses ASes, each AS adds its own mark to the packet's header. By the time the packet reaches its destination AS, the marks placed in its header form a signature unique (as much as possible) to the AS-path it has taken. These path signatures can be used to perform IP traceback by comparing the path signature of an incoming packet with previously acquired path signatures whose origin ASes are known ${ }^{1}$. In this paper we propose and evaluate two methods for comparing signatures when

\footnotetext{
${ }^{1}$ Path signatures can also be used to filter out attack packets originating from the same source and to identify a packet as spoofed if the path signature it contains does not match the one expected of the source it is claiming to be coming from.
} 
performing IP traceback.

\subsection{Routing Infrastructure Considerations}

\subsubsection{Secure BGP Routing Environment}

The Border Gateway Protocol (BGP) [46] is the standard inter-AS routing protocol used between ASes to discover and maintain routing information in order to guide traffic across the Internet. The current routing infrastructure is vulnerable to a number of malicious attacks due to a lack of a secure means to verify the authenticity and integrity of BGP control messages. For many years now researchers have been coming up with proposals to address the security issues in the BGP routing infrastructure. Some of the notable approaches proposed include S-BGP [33], so-BGP [56], and psBGP [54]. For an informative summary of these three proposed systems, the reader is referred to [55].

Another approach to secure BGP, which we would like to briefly discuss here also, is called Interdomain Route Validation (IRV) [29]. Compared to the other three approaches mentioned earlier, IRV is more geared towards incremental deployment. It is a new protocol separate from BGP, which serves as a companion to it. IRV is receiver-driven in the sense that it enables the receiver of BGP information to communicate with the sender and corroborate the information. Each AS has an IRV server, which can communicate directly with the IRV server of another AS over a 
secure channel to corroborate information it may have sent. Similarly, in our approach each AS has a dedicated server for our solution, which can communicate directly with the dedicated servers of other ASes.

Our approach requires that a secure inter-AS (BGP) routing infrastructure be present so that ASes deploying our solution can reliably learn which IP prefixes are assigned to which ASes. This infrastructure would also be used to exchange authenticated messages in order to associate path signatures of packets with their true source ASes. We assume the eventual adoption of a secured BGP routing infrastructure to combat other serious security issues on the Internet, and to facilitate a multitude of security services in IP network. Based on this we believe our assumption to be reasonable. For our purposes we can assume that whichever method is eventually implemented, it provides each AS with a public key certificate whose corresponding private key can be used to sign outgoing messages.

\subsubsection{Using BGP to Transmit Capability Information}

BGP speaking routers regularly exchange routing information using BGP update messages. These update messages may contain a number of path attributes. The optional extended communities path attribute [48] can be used to pass additional information to both neighbouring and remote BGP peers, and thus create a logical grouping of ASes that share common characteristics.

We propose the use of the transitive BGP extended communities path attribute 
in order for ASes to learn which other ASes also support our solution in a partial deployment scenario with legacy ASes present. This should aid the incremental deployment of our solution in an environment where not all ASes will initially support it. The extended communities path attribute would be distributed as part of outgoing BGP update messages being sent out by ASes that support our solution. The transmitted BGP updates with this new path attribute would lead to the creation of a logical community within the network of interconnected ASes that support our solution. Legacy ASes are not a problem in this approach because they are expected to pass along an unrecognized transitive path attribute without any modification.

\subsubsection{Encoding Format for the Extended Communities Path Attribute}

A BGP extended communities attribute, as illustrated in Fig. 3.1, is 8 bytes long, and consists of a type and value fields. The type field can be either one or two bytes long, while the value field would occupy the remaining bytes. The most significant 4 bits of the first byte in the type field constitute flags. We would need a new unique BGP extended community type to be allocated by IANA [2] for use by our solution. An AS supporting our solution that is sending out a BGP update with an extended communities path attribute in it, would set the type field of the path attribute to the IANA [2] assigned value for our solution, and would set the value field to the IP address of a server within this AS that is responsible for running tasks specifically related to our solution. The responsibilities of this server will be further discussed in 
section 3.7 .

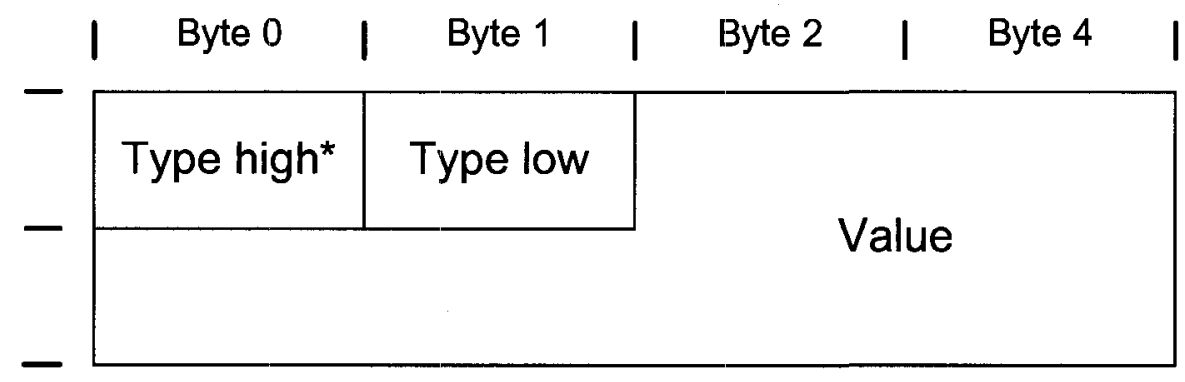

${ }^{*}$ Most significant 4 bits are flags

Figure 3.1: Structure of the BGP extended communities path attribute

There is a complication with the approach described here to transmit capability information. A BGP path attribute can be variable length and occupy many bytes. However, the structure of the extended communities path attribute has been specifically defined to be constant in length and limited to only 8 bytes. Eight bytes is long enough to accommodate a 4-byte long IPv4 address in the value field, however it is not long enough to accommodate a 16-byte long IPv6 address. Since the extended communities path attribute is in principle designed to achieve the same type of objectives we are after here, it is unwise to abandon it and require an entirely new kind of BGP path attribute be defined for our solution.

It should be possible to work around the 8-byte length limitation in the extended communities path attribute. A BGP update message can contain a variable number of path attributes. Therefore, we simply propose using two extended communities path attributes in a single update message to convey the IPv6 address of the sending AS' server responsible for performing functionality specific to our solution. Overall, 
we would need four extended community types assigned by IANA as follows:

1. Used in an IPv4 environment

2. Used in an IPv6 environment; contains the first part of the server's IPv6 address

3. Used in an IPv6 environment; contains the second part of the server's IPv6 address

4. Used in an IPv6 environment; contains the third part of the server's IPv6 address

It is also possible to get by with just three extended community type values by making use of the Partial bit flag. As previously mentioned, the most significant 4 bits of the first byte in the type field are flags. The third high order bit of these flags is the Partial bit flag, which defines whether the information in the optional transitive path attribute is partial or complete. Our first IANA assigned extended community type can be interpreted to be paired with an IPv4 address if the partial bit is set to 0 , and be interpreted to be paired with the first part of an IPv6 address if set to 1 .

\subsection{Finding Storage Space Inside of an IP Packet}

Each AS along the path needs to insert a small amount of information in the packet's header. IP was not designed with such a requirement in mind, at least not for realtime forwarding of a large number of packets. Using IP options for this purpose is not feasible because it significantly slows down processing speed and can lead to 
fragmentation or dropped packets as a result of increasing the packet's size. Savage et al. addressed this problem in [49] by overloading the rarely used 16-bit IPv4 identification field normally used for fragmentation. It should be noted that this field is initialized to some random value before transmission by the sending node's operating system.

Since we are already breaking fragmentation by overloading the IP identification field as is done in many IP traceback approaches, we looked into the possibility of using the remaining fragmentation bits in the IP header while minimizing any negative impacts this would have. Most approaches do not use the remaining fragmentation bits, and those that do, do not address the adverse effects it would have. Fig. 3.2 illustrates the format of the IPv4 header, and should help the reader better visualize what we are about to discuss. The three flags in Fig. 3.2 are as follows:

- R: Reserved

- DF: Do not fragment

- MF: More fragments

Current approaches do not use the remaining bits because it may cause some networking stacks, such as the Linux 2.6 [11] network stack, to mistake marked packets for fragmented ones. We examined the source code for kernel version 2.6.26 specifically, to see how it determines if an IP packet is a fragment or not. This checking is done in ip_input.c, in function ip_local_deliver(...). A packet is considered to be a fragment: 


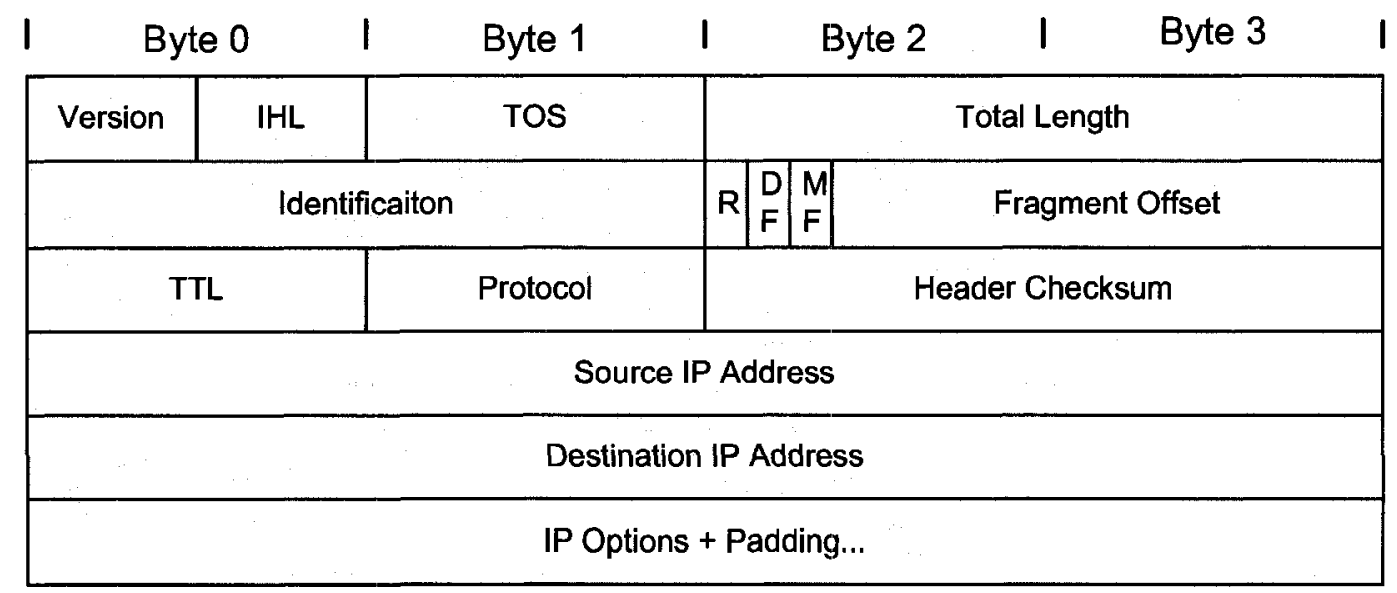

Figure 3.2: IPv4 header

1. If this is the last fragment ( $M F=0 \mathrm{AND}$ Fragment Offset is non-zero)

2. If more fragments follow this fragment $(\mathrm{MF}=1)$

What we observed was that the code was not checking the value of the Do not fragment $(D F)$ flag. Logically if the DF flag is set, the packet should not be treated as a fragment, no matter what the values of the MF flag and the fragment offset field are. Of course in normal IPv4 operation there should be no situation in which DF is set while the other two variables indicate a packet fragment.

We believe we can use the Reserved flag, which is currently unused, as part of the marking field in our marking scheme without any issues. Should it ever be needed in the future as part of a specific feature, this one bit will not significantly impact our results.

We can use the DF flag to propose a very minor change to the stack code where a packet is not treated as a fragment if this flag is set. Checking this additional flag 
when deciding if a packet is a fragment or not is trivial. This should let us use as much as 31 bits of the fragmentation fields ( $32^{\text {nd }}$ bit used for Do not fragment) and allow for both more bits per AS mark and more marks in the packet. We tried both 16-bit and 31-bit marking fields in our simulations.

While we were able to confirm that the Linux kernel networking code does not check the DF flag when deciding if a packet is a fragment or not, we do not have access to the source code of other operating systems such as Windows, or the Cisco IOS, therefore we do not know if such behaviour is common across all networking stacks. As already mentioned, the code change is trivial, but it would involve patching all legacy networking stacks on the Internet if our solution were to be put in place with 31-bit marking. Otherwise, unpatched destinations would interpret our marked packets as fragments and would be unable to receive traffic properly.

We propose a possible work around for the problem of unpatched destinations interpreting marked packets as fragments. At the edge of the destination AS, after confirming that the packet has not been spoofed based on our marking strategy, the edge router can overwrite our mark by setting the MF flag to 0 , and the Fragment offset field to 0 , thus preventing any unpatched recipient node of the packet in that AS from treating it as a fragment. This would work for an AS that supports our solution and is aware of our marking scheme, however it would not work for a legacy AS. Another possible solution can be that packets are only marked if their final destination AS supports our solution. The issue with this approach would be the 
Design and Architecture of the Hierarchical IP Traceback System

overhead it would add to intermediate edge routers to check the destination IP of every packet and see if it maps to an AS supporting our solution. This remains an open research question.

\subsection{Marking Packets - Simple Strategy}

This section explains how we perform packet marking (Fig. 3.3) where we assume all ASes deploy our solution. In Section 3.5 we deal with the more complex case where some ASes do not have our solution deployed (we call them legacy ASes). Each AS uses part of an MD5 [47] hash of its AS number as the mark it places in the header of packets traversing it. We chose the widely used MD5 cryptographic hash function because its speed and ease of use. We are aware of collision attacks on MD5, however since we are not using it for signing purposes, these attacks do not affect us. The length of the mark in terms of the number of bits each AS gets to use would be constant across the entire Internet. This length should be chosen so as to give as much uniqueness to the mark as possible, while allowing enough marks to fit into the IP header field to properly represent the length of the path. In Section 4.1.1 we discuss how we chose this length.

Packet markings start at the egress AS router where the packet originated, followed by the ingress router of every AS that the packet traverses except the destination AS. This will result in a path signature to form in the packet by the time it reaches the destination AS. 


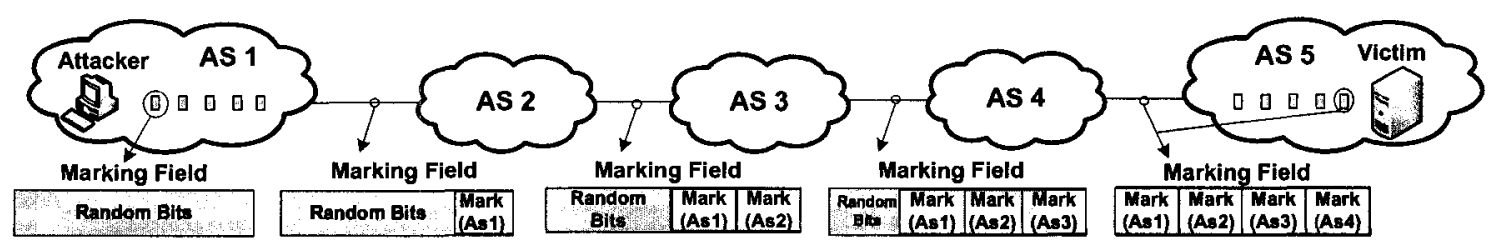

Figure 3.3: Basic marking scheme at the AS-level

Each router shifts the bits in the marking field to the left by $n$ bits before placing its $n$-bit long mark in this field at the rightmost position. Each time the packet is marked, $n$ bits of the original marking field value are dropped and replaced with legitimate marking bits. If there are more ASes along the path than the number of marks which can be accommodated in the marking field, then only the later ones will be retained in the path signature.

\subsection{Marking Packets with Support for Incremental}

\section{Deployment}

To fulfill incremental deployment, our solution can support legacy ASes (those that do not deploy it). To support this, we need to change both the marking strategy as well as how path signature id reply messages are sent (discussed in Section 3.7). We should note that for a destination AS to use the features discussed here, such as IP traceback, it must have our solution deployed (not be a legacy AS).

As shown in Fig. 3.4, instead of an AS' mark being based on the hash of just its own AS number, it is now based on the links between ASes. As well, ASes now have 
to be more proactive to compensate for legacy ASes not putting in their own marks. When an AS marks an outgoing packet, it will mark it with the MD5 hash of its own AS number concatenated with the AS number of the next AS along the path. When an AS receives a packet, it expects the most recent mark to be that of the incoming link the packet arrived on. If this is not the case then it will insert the corresponding mark into the packet itself.

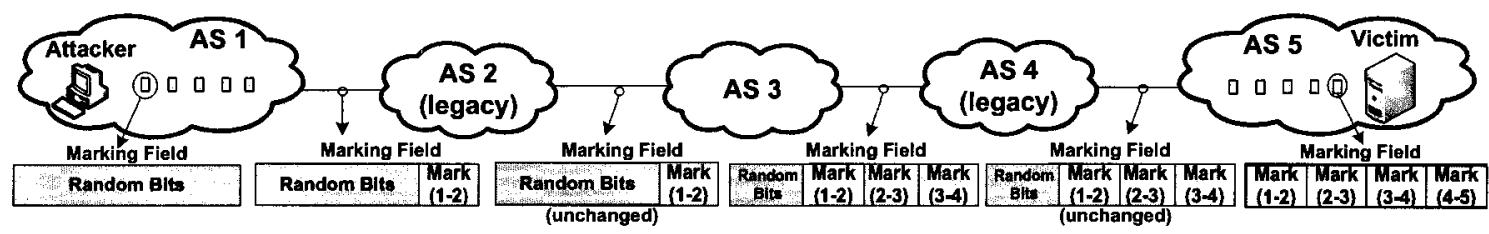

Figure 3.4: Marking scheme with legacy AS support

To support legacy ASes, when sending out path signature id reply messages, an AS should also send out ones on behalf of its neighbouring legacy ASes. We will discuss this further in Section 3.7.

\subsection{Further Enhancements to Our Marking Strat-}

\section{egy}

The two marking strategies presented so far are AS-level implementations which are similar to the marking strategies put forth by the StackPi researchers at the routerlevel. Given that we are working at the AS-level, we can make further enhancements to the previously discussed strategies which are not possible at the router-level. 
Having the marks be based on well known values such as AS numbers does pose a security risk. An attacker wanting to spoof path signatures would know what legitimate path signatures between ASes would look like. To avoid this, the marks should be based on, or influenced by, a secret known only to the marking AS. This way the attacker would no longer know what another AS' path signatures look like, not without being able to sniff traffic on the backbone links, in which case this would signal a much more serious security problem than IP spoofing. One simple way of achieving this new requirement would be that an AS picks a secret key, and sets the mark on each of its links to other ASes to be the MD5 hash of this key concatenated with the AS number of the AS on the other side of the link. Previously the mark was based on the MD5 hash of this AS' number concatenated with the AS number of the AS on the other side of the link.

Marking links at an AS based on a secret key requires another change to the overall marking strategy. Previously, we required that when an AS receives a packet, it would expect the most recent mark in it to be that of the incoming link the packet arrived on. If this was not the case, then this signalled the fact that the AS on the other side of the link was a legacy AS and hence this AS would compensate by inserting the corresponding link mark into the packet. With link marks that are based on a secret key, a downstream AS would not know what mark to expect from the upstream AS. This is the reason why this approach cannot be taken at the router level, at least not without a lot of overhead. However, at the AS-level due to BGP advertisements an 
AS can learn which of its neighbours are legacy ASes. Therefore, in this iteration of our marking strategy an AS supporting our solution would no longer need to inspect the most recent mark in an incoming packet, if it was forwarded by an upstream AS that also supports our solution. It is guaranteed that the packet was marked by the upstream AS. If packets are arriving on a link that is connected to a legacy AS, then this AS should automatically mark the packets. Hence, no AS should need to know the secret key being used by another AS to mark packets.

\subsection{Associating a Path Signature with its Source}

\section{AS}

At the victim AS, before an incoming packet can be checked to see if it is spoofed or not, two pieces of information are needed:

- The expected origin AS for the packet's source IP address

- Expected path signature for this origin AS

Each AS supporting our solution should designate a server to exchange messages with other supporting ASes, and maintain information specific to our solution. This AS would disseminate the address of its server to other ASes through BGP updates, as was discussed in Section 3.2.2.1. The server will maintain communication with all the border routers of its AS and provide them with the information they need to 
carry out our solution.

The first responsibility of the server is maintaining a mapping of IP prefixes in use on the Internet to their corresponding AS numbers. This can be done offline. There are already groups on the Internet such as [4] which monitor BGP advertisements using several distributed BGP speaking routers and provide this type of functionality.

Learning which path signature belongs to which AS is the main responsibility of the server. The server in each AS would maintain a database of the mappings between origin ASes and their expected path signatures as it learns this information.

\subsubsection{Learning AS-Path Signatures}

Our research was primarily concerned with IP traceback. Hence, we used a simple signature distribution mechanism for our simulations, as explained in Section 4.3.2. Nevertheless, here we propose two candidate mechanisms by which an AS can learn what path signature to expect when receiving packets from a certain origin AS. Testing, and fine turning these two mechanisms is the subject of future research. The two mechanisms we propose are as follows:

1. On-demand request directed to the origin AS

2. Proactive periodic sending of path signatures by upstream ASes 


\subsubsection{On-Demand Request Directed to the Origin AS}

In the first mechanism, if a destination AS receives a packet and its server doesn't have a path signature for the packet's claimed origin AS, the destination AS server would send a path signature id req message to the origin AS server. This request would be signed using public key cryptography to confirm the requester's identity. The origin AS server would send a reply packet whose data field it would sign using public key cryptography so that the recipient can validate the sender's identity. For both the request and reply packets, in addition to signed information about the identity of the sending AS, the packets should also contain a digitally signed times-tamp. The purpose of this timestamp is to prevent a replay attack. Other means of combating a replay attack could also be used instead, such as session tokens. The request and reply sequence is shown in Fig. 3.5. Beyond this, the details of the protocol are more of an engineering problem and not the focus of this research.

The cryptography used would be facilitated by the secure BGP routing architecture requirement we previously discussed in Section 3.2.1. Authentication through digital signatures should be adequate for most situations, however if confidentiality is also desired, the same secure BGP routing architecture can provide for encryption as well. The reply packet would travel on the exact same path taken by normal packets being sent from this AS, thus ensuring that the proper path signature is learned even in the presence of asymmetrical routing.

Actually, we propose sending multiple reply packets to the destination AS server. 
This is because if the source and destination ASes are close enough to each other in terms of intermediate ASes, then not all the random data the IP identification field was initialized with would have been pushed out of it by the time it reaches the requesting AS. By sending multiple reply packets, the destination can compare the values in the identification fields of these packets and only interpret the bits constant across all the packets as being the unique path signature. In Section 6.4 we discuss how many packets were needed to be sent in our simulations.

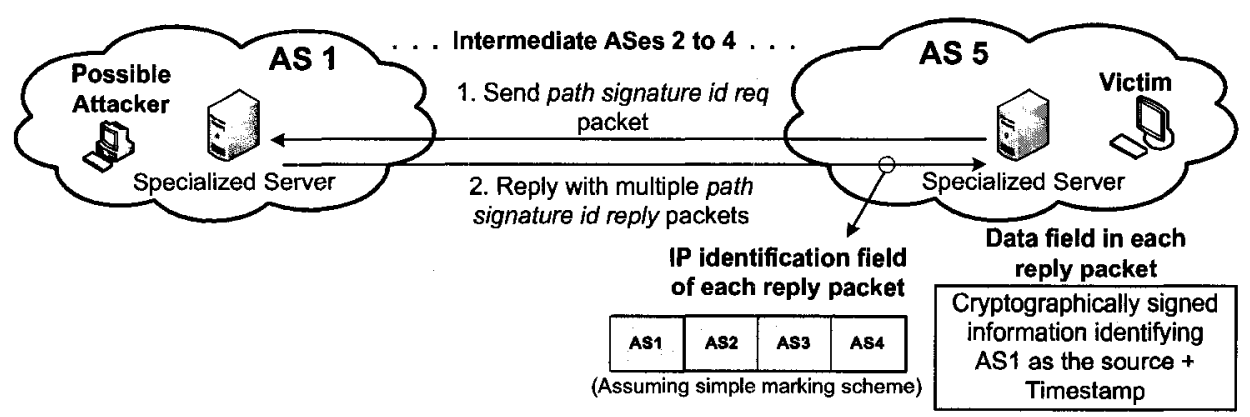

Figure 3.5: On-demand request for path signature by AS 5 from origin AS 1 (assuming simple marking strategy)

\subsubsection{Proactive Probabilistic Path Signature Generation Methodology}

The second mechanism for learning path signatures was inspired by the work done in iTrace [16], and the improvements done to it in [39]. Our solution builds upon both works. If we were to adopt basic iTrace, this would involve an AS forwarding traffic to other ASes to sample every $n^{\text {th }}$ packet ( 1 in every 20,000 was chosen in iTrace) and proactively send path signature id reply packets to the sampled packet's destination AS. There is much room for improvement here. Firstly, an AS sending these packets 
should only send them to a destination it knows supports our solution, as stated in [39]. As previously discussed, an AS learns which others support our solution through BGP advertisements. An AS should remember which other destination ASes it has recently sent path signature id reply packets to and avoid sending them such packets again. This would be wasteful since AS-paths are relatively stable and do not change often [20]. We will refer to the minimum time delay between sending path signature id reply packets to the same destination AS as $t$ time units. $t$ can be selected to be a relatively large value, in the order of several days. Fine-tuning this value can be the focus of future work.

The details of our second mechanism for learning path signatures are as follows:

- Dedicated server maintains a list of all ASes it has learned support our solution

- Dedicated server will keep a record of when it last sent path signature id reply messages to an AS

- Every $k$ time units, the dedicated server will send out path signature id reply messages to an AS, which is chosen as will be explained shortly

- The proper value for $k$ is the subject of future work

- Edge routers sample every 1 in 20,000 packets, extract the destination IP and forward it to the dedicated server using a new protocol as part of our solution

- The purpose of this is to build a list of "recommended" destinations at the dedicated server 
- These "recommended" destinations are ones that the current AS forwards a lot of traffic to, and are better candidates for receiving path signature id reply messages than a randomly chosen AS

- The dedicated server can map the destination IP address to the corresponding destination $\mathrm{AS}$, and verify that it supports our solution, so as to lessen the workload on the edge router

- The 1 in 20,000 ratio was chosen based on iTrace work, but can be finetuned in future work

- At every $k$ period, the server will first pick randomly from the list of "recommended" AS destinations to send path signature id reply messages to, as long as it hasn't already sent that destination such messages within the last $t$ time units

- If the server can't find an eligible destination AS in the "recommended" AS list, it will randomly pick one from the full list of destination ASes supporting our solution (similarly, it must not have been sent messages within the past $t$ time units)

- $t$ is the minimum time delay between sending path signature id reply packets to the same destination $\mathrm{AS}$

- $t$ can be selected to be in the order of several days, based on research cited earlier which indicates AS-paths are highly stable 


\subsubsection{Path Signature Generation on Behalf of Legacy ASes}

In our previous discussion, when an AS generates path signature id reply messages triggered either by an on-demand request, or periodically, these messages only convey this AS' path signature to the target AS. This is fine in an ideal situation where there are no legacy ASes present. However, since any realistic solution has to support legacy ASes as well, we require some additional work from each solution-supporting AS to convey path signature id reply messages on behalf of their neighbouring legacy ASes as well.

In Fig. 3.6 we show how a solution-supporting AS would send out path signature id reply messages on behalf of its neighbouring legacy ASes as well when sending out its own. The dedicated server in AS 2 would need know what marking signature is used on the links between AS 2 and each of its neighbouring legacy ASes. All 3 packets shown are generated by the server. The first packet corresponds to the path signature for AS 2 itself, while the bottom two correspond to each of the legacy ASes. When generating each of the bottom 2 packets, the server pushes the corresponding mark for the link to the legacy ASes into the marking field of the packets.

There are a number of important considerations to keep in mind however beyond the simple topology shown in Fig. 3.6. If a legacy AS has no neighbouring ASes that support our solution, then there is no way to learn a unique signature for this AS at the target AS. Even if a legacy AS has a supporting neighbour that generates a signature on its behalf to a target AS, this legacy AS might be multi-homed to 


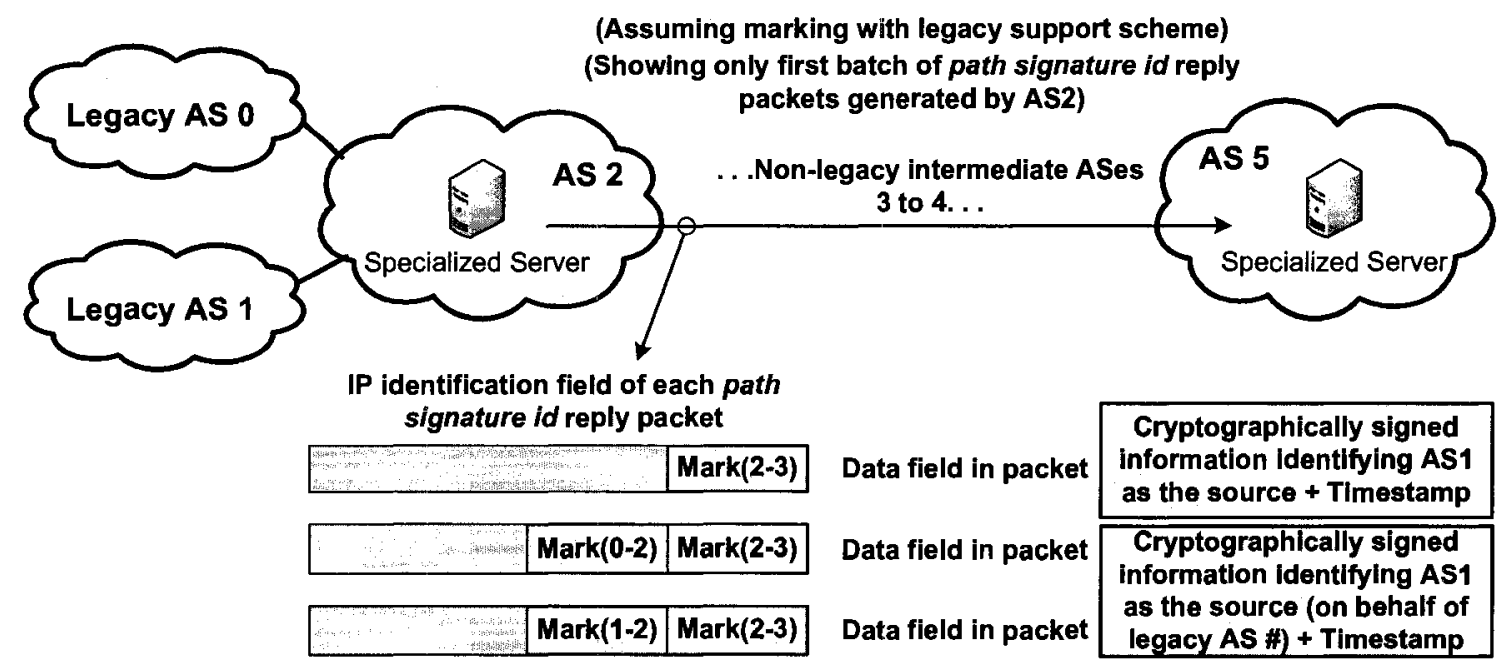

Figure 3.6: Path signature generation by a supporting AS on behalf of its neighbouring legacy ASes

another legacy AS which it uses to send traffic to the target AS. In this case packets arriving at the target AS from the legacy one will not contain the path signature learned.

When learning the path signature of a legacy AS through another solution-supporting AS, the target AS should try to verify the learned signature after receiving live traffic from the legacy AS. One option is to assume the live traffic to be non-spoofed during a set bootstrapping time, if it passes through IDS checks, etc. Alternatively, the live traffic can be verified to be non-spoofed through the TCP Intercept [9] router feature for example.

We should also comment on the on-demand mechanism for learning path signatures in the presence of legacy ASes. Through publicly available data, such as [6], it is sometimes possible to identify the neighbouring ASes of a given AS. A target 
AS that is interested in learning the path signature of a legacy AS can identify its solution-supporting neighbours, if any, and ask them for the signature of that legacy AS. Once again we should note that there is no guarantee the legacy AS is forwarding traffic through any of these solution-supporting ASes.

\subsubsection{Single-Homed and Multi-Homed Customer Considera- tions}

In the context of associating a source AS to its path signature, we will discuss how customer networks are connected to their service provider (ISP), and whether or not every customer network has its own individual AS number. Two RFCs [31], [52] deal with the issue of when it is appropriate to create a new AS with its own globally unique AS number. In Fig. 3.7 we give examples of possible connections between a customer network and its ISP. In this example, customer network 1 is multi-homed to a single ISP, customer network 2 is multi-homed to two different ISPs, while customer network 3 is a singly-homed stub network.

The previously mentioned RFCs state that an AS should only be created if its routing policy (how the rest of the Internet should forward traffic to it) is different than its provider. Customer Network 2, which is multi-homed to two different ISPs, should acquire a globally unique AS number of its own to use. For such a network, in our solution, at the destination AS we would need to map two different possible path signatures to the same AS number for Customer Network 2, assuming it is actively 


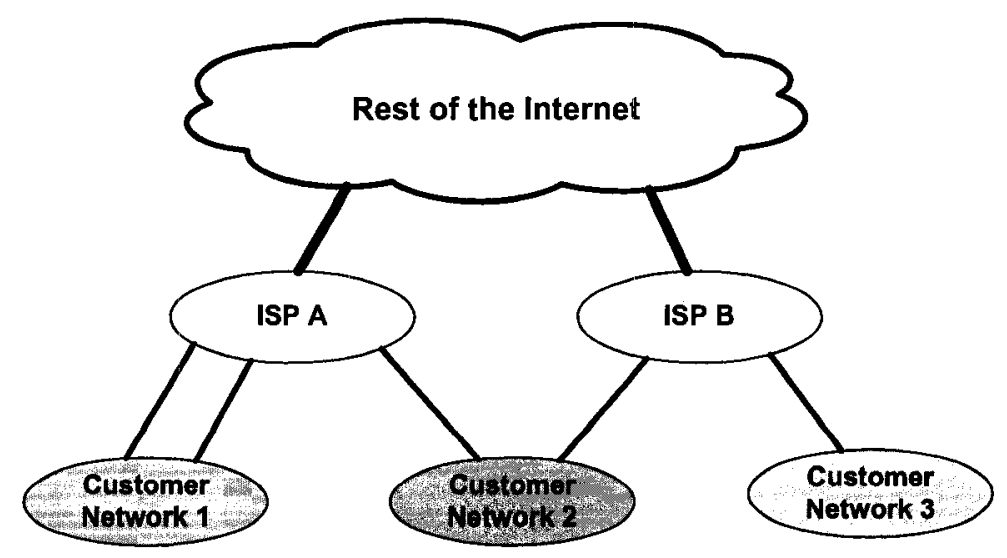

Figure 3.7: Examples of customer network connectivity to ISP

using both links at the same time (load sharing).

In the cases of customer networks 1 and 3, they are connected to a single ISP, and therefore from the perspective of the rest of the Internet, traffic is directed to them the same as it would be for their ISP. Essentially they share the same routing policy as their ISP, hence the RFCs state that they should not acquire their own globally unique AS numbers. RFC 2270 [52] offers two options in this case:

1. Be assigned a private AS number (range 64512 to 65535 ) by ISP

2. Use an AS number previously allocated to ISP

For the first option, the private AS number used by the customer network would be stripped out of BGP messages leaving the ISP. Private AS numbers are on some level similar to non-routable IP prefixes like 10.0.0.0/8. In our solution, the destination AS would only see the AS number of the ISP. However, this does not necessarily mean that we cannot allow a different path signature for the customer networks than their 
ISP. We can have different path signatures associated with this ISP's AS number, one for packets originating from within the ISP (if any), and another path signature per customer network. This can be achieved because there is a distinct edge between the ISP and each customer network, at which point a mark associated with this link can be placed in the marking field of the packets. When the dedicated server in the ISP is sending out its path signatures, it will simply transmit multiple ones for itself to the destination AS. This isn't particularly useful in terms of enhancing the detection of spoofed packets, because at the destination we wouldn't have information about which part of the IP prefix announced by the source ISP is used by which of its customer networks. However, it is useful when performing IP traceback, since based on which signature the attack packets contain, law enforcement would be able to inquire from the ISP as to which of its customer networks the path signature belongs to. This would also be useful in filtering attack traffic, because if only a specific customer network is responsible, its signature should be different from the other networks present within that same source AS, hence instead of filtering traffic coming from the entire ISP we can narrow down filtering to those packets originating from the path signature of the offending customer network.

Regarding the second option where the customer networks use an AS number previously allocated to their ISP by IANA, what we discussed for the case of private AS numbers should also apply here. In both cases, only the ISP network itself should run a server dedicated to our solution. Since the AS numbers used by all the customer 
networks and the ISP may be the same, the marking would have to be based on the scheme we discussed in section 3.6.

\subsection{Spoofing Detection and Other Security Con-}

\section{siderations}

Once the path signature of an origin AS is learned, and verified if it belongs to a legacy AS, spoofed packets can be detected by simply comparing the path signature of incoming packets against the expected one.

An excellent classification of various ways to spoof source addresses is provided in [41]. Address routability, whether the spoofed source address can be routed to or not, should not have any impact on our technique. Spoofing technique, how the attacker chooses the spoofed source address in attack packets, deserves some discussion. A subnet spoofed source address, where the attacker spoofs a random address from the address space assigned to the attacking machine's subnet, would not be detected by our approach. It is in fact impossible to detect this between the subnet's exit router and the victim machine. To generalize this attack vector a little further, if the spoofed address is within the legitimately assigned IP prefix for that AS, we cannot detect it with our approach. This is because our approach works at the AS level and does not have the fine grained control and monitoring of the sending AS' internal network required to detect such an attack. The contribution of our approach in such a case 
would be that once the attack packet is identified as being malicious at the victim network, we could perform IP traceback on it and narrow down the ASes it may have come from.

Another interesting attack vector is enroute spoofing, where the attacker spoofs the address of a machine or subnet that lies along the path from the agent machine to the victim. Our solution would be susceptible to this type of spoofing. However, once an attack packet with this type of spoofing is detected as being malicious at the victim, we can perform traceback on it. Since the attack packet would contain the full marked path from the real source to the victim, traceback should be reasonably effective.

There is a limitation to our approach imposed by the total number of marks that can be accommodated in the marking field. An attacker in AS X can implicate a node in AS Y, if packets from both ASes converge on the same AS-path towards a victim in AS Z, given that both ASes X and $\mathrm{Y}$ are distant enough from AS Z such that their learned path signatures at $\mathrm{AS} \mathrm{Z}$ corresponds only to the portion of the path shared by both ASes $\mathrm{X}$ and $\mathrm{Y}$. The more marks that can be accommodated in the marking field, the more resilient our approach can be to such attacks.

\subsection{Identifying the Origin AS (IP Traceback)}

The origin AS can be identified by matching an incoming packet's path signature against all the known AS-path signatures in the database of the target AS' server. 
There are two ways of doing this as shown in Fig. 3.8.

\section{Longest Matching Signature (LMS)}

Path signature in packet:
$\mathbf{1 0 1 1} \mathbf{1 1 0 0} \mathbf{0 1 1 0} \mathbf{0 1 0 0}$
Known AS Path Signatures:
1011110001100101
101111000111
101111000110
10111100
10110100
1011

\section{All Matching Signatures (AMS)}

Path signature in packet: 1011110001100100

Known AS Path Signatures: 1011110001100101 101111000111 101111000110 10111100 10110100 1011

Figure 3.8: Path signature matching methods for IP traceback

If the path signature of the origin AS exists in the server's database, the all matching signatures (AMS) approach will select it along with signatures of intermediate ASes and possibly a few other incorrect ASes whose signatures may have randomly ended up being (due to limited number of bits to represent AS marks) the same as ASes that are of interest to us. In the same situation the longest matching signature (LMS) technique will in most cases select the correct origin AS-path signature. It should be noted that a single AS-path signature in the server database may map to multiple different ASes due to limited number of bits per mark, or limited number of total marks that can be accommodated in the marking field. If the server's database doesn't contain the path signature of the origin AS, the AMS and LMS techniques are still likely to produce a list of potential source ASes whose path signatures are similar to the path signature in the incoming packet.

Our approach would work best if paired with another IP traceback system local 
to each AS. Once we identify one or more ASes as the potential source of a spoofed packet, we can launch parallel queries to the local IP traceback system of those ASes and determine which of them actually sent the packet. We consider a packet loggingbased approach as a good candidate for the local IP traceback system at each AS. This is because our approach stores information in the packet header, which would conflict with other packet marking-based approaches. However, logging-based approaches don't store information in the packet header and therefore would not conflict with ours.

Given enough time for an AS running our system to learn path signatures of other ASes, IP traceback can be carried out on single packets. Since our hierarchical solution works to identify the source AS rather than the sending node itself, issues such as NAT within that AS would not hinder our approach.

IP Traceback using our solution is not limited to "live" incoming packets. It can also be performed on packets previously captured and logged. This would facilitate forensic investigations by allowing the path signature of logged packets to be checked against either the current known database of path signatures, or more appropriately a captured image of path signatures which were known around the same time as when the packet was logged. On this note, it would serve as a useful feature to have the dedicated server which is learning path signatures store an image of these signatures every $t$ time units to allow for more accurate forensics on logged packets. Based on the findings in $[20], t$ can be selected to be a relatively large value, in the order of 
several days, due to BGP path stability. 


\section{Chapter 4}

\section{Simulation Models and}

\section{Methodology}

\subsection{Topology Generation}

In order to generate a reasonable representation of the actual AS topology in the Internet to test our solution with, we first looked at Inet3 [57]. Inet3 is a prominent AS-level topology generator that makes use of power laws.

According to [1] there are currently about 50,000 AS numbers assigned. Therefore, we chose to base our simulations on a topology with 5000 ASes. This amounts to $10 \%$ of the currently assigned ASes, which should be large enough to make a reasonable representation of the Internet, and at the same time small enough so that each simulation completes in a reasonable time. We used the default settings in Inet3 
to generate a topology with 5000 ASes. In order to analyze a generated topology and derive statistics such as the mean degree and mean distance, we used a tool called Network Manipulator (nem) [38].

Analyzing the generated topology revealed the overall mean degree and mean distance to be 3.48 , and 3.54 ASes respectively. Another variable of interest is the maximum degree, which was found to be 1026. In Fig. 4.1 we illustrate a histogram of the mean distances of this topology, and in Fig. 4.2 we illustrate a histogram of the AS degrees. The mean distance holds significance in terms of how many total marks we should select to be recorded in the packet, while the degree statistics are to be considered when choosing how many bits are allocated to each AS mark. For example, if we were to allocate 3 bits per AS mark, it would mean that this AS could potentially come up with 8 unique signatures for its neighbours. If we were to find out that a significant number of ASes have more than 8 neighbours, then a higher number of bits per AS mark would be appropriate. 


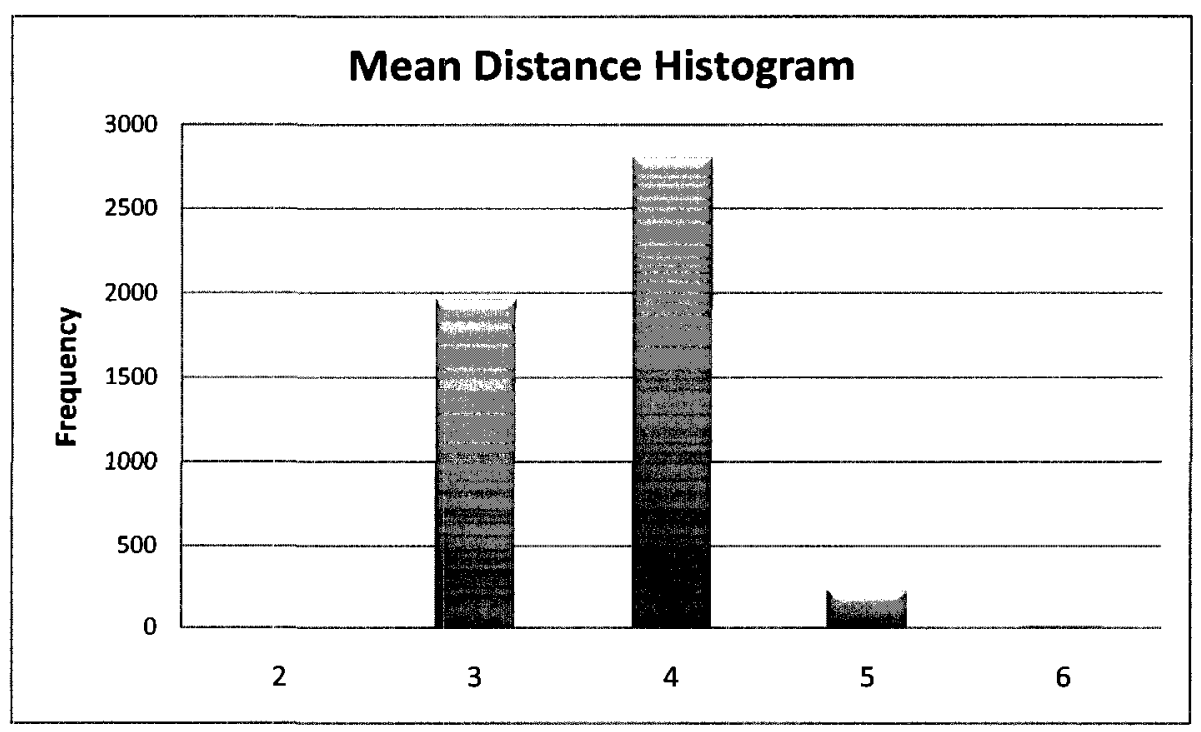

Figure 4.1: Histogram of mean distances in the Inet3 topology

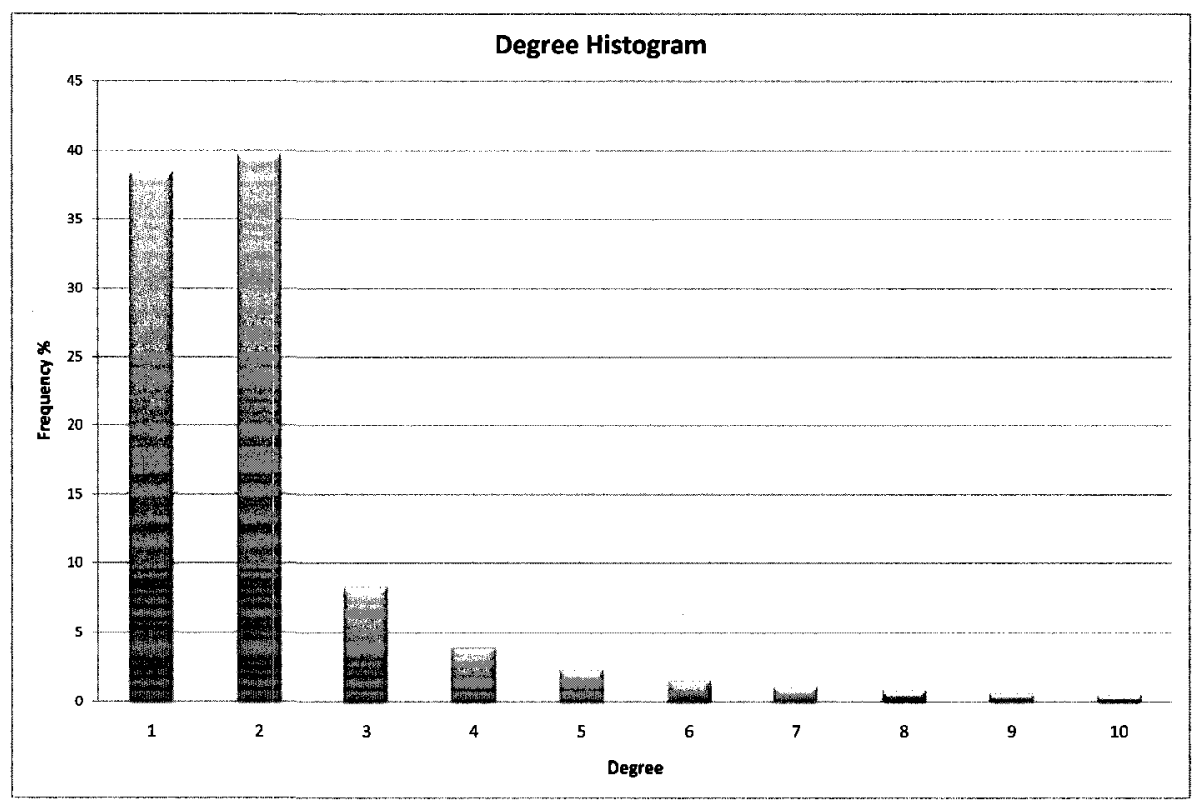

Figure 4.2: Histogram of $\mathrm{AS}$ degrees in the Inet3 topology (only showing up to a degree of 10)

We considered the Inet3 generated topology's mean distance to be a good starting 
point for choosing the number of AS marks to record in each packet. Looking at Fig. 4.2, almost all the ASes have just one or two neighbours. This alone would suggest that we need very few bits allocated to each mark. However, we found that there are a few ASes in this topology that act as major carriers and hence have a very high degree. This is to be expected with power law based topology generators. As noted earlier, this topology has a maximum degree of 1026 for one of its ASes. Since so many ASes are connected to these carrier ASes, it is best to allocate as many bits as possible to each AS mark so that the marks used by these carrier ASes are as unique as possible. There will of course be a significant amount of overlap in the marks these carrier ASes use, but we will show that we can still achieve good accuracy in our results.

Based on this information we chose to allow up to 4 marks to be placed in a 16-bit marking field, where each AS mark would be 4 bits long. This was only for the initial test case. As will be shown later we have run many other test cases with differing number of total AS marks, and different number of bits per mark.

\subsubsection{Average AS Path Length}

The average AS path length on the Internet is an important metric to understand in order to decide how many bits in the marking field should be allocated to each AS mark so that a useful number of ASes get a chance to place their marks in this field. We looked at studies of the average AS-path length on the Internet which suggest 
path lengths such as $5[5]$ or $6[3]$ ASes are typical of the Internet. The reason why topologies generated with Inet3 have shorter average AS path lengths might be that Inet3 generates topologies with characteristics similar to Internet topologies from 1997 to 2002 [57]. Looking at the topology analysis done in [57], it seems back then the Internet average AS path length was shorter than what it currently is. To this end we looked into obtaining another topology for simulation with a longer average path length.

Using the Brite [40] topology generator we generated a second AS-level topology with 5000 ASes. Analyzing this topology revealed the mean degree and mean distance to be 4 , and 4.76 respectively. The maximum degree was found to be 228 neighbouring ASes. Figures 4.3 and 4.4 illustrate histograms of the mean distances, and AS degrees for this topology.

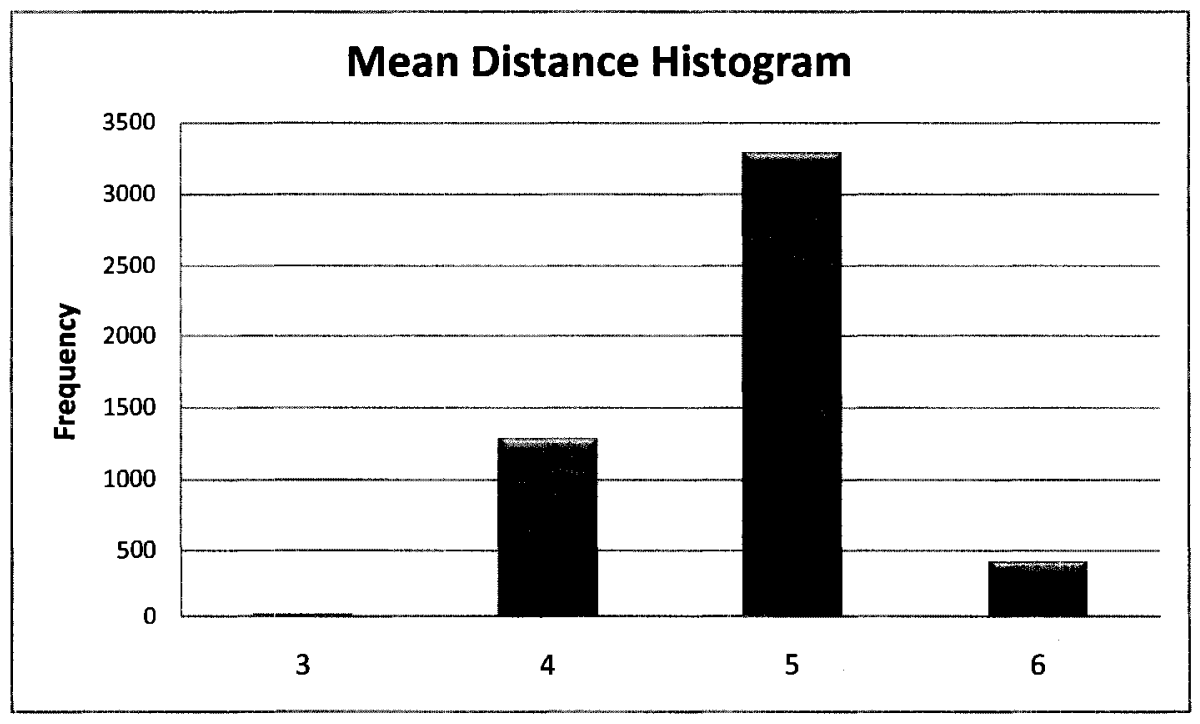

Figure 4.3: Histogram of mean distances in the Brite topology 


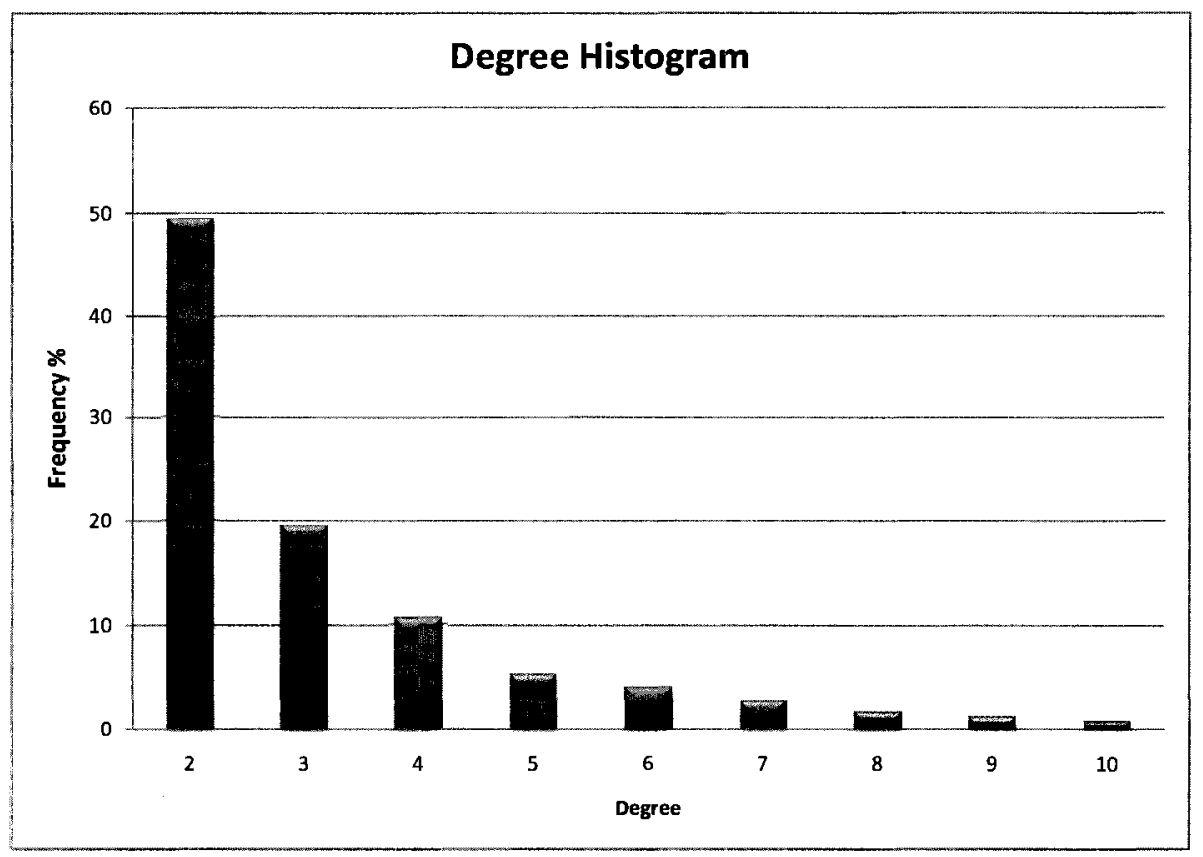

Figure 4.4: Histogram of AS degrees in the Brite topology (only showing up to a degree of 10)

\subsection{NS-2 Modifications}

In order to evaluate our solution, we ran numerous simulations using the discrete event network simulator ns-2 [7]. Since ns-2 is open source and is well documented, it is relatively easy to extend. For these reasons ns-2 is very popular in academia. Here we will discuss the main modifications we made to the ns-2 codebase in order to implement those aspects of our solution which we tested using simulations. In Appendix A we will provide a list of all the files modified, as well as the specific reasons behind these modifications. 


\subsubsection{Traffic Agents}

An Agent object in ns-2 is an endpoint responsible for either constructing packets, or consuming them. We created two new agents. The first, which was responsible for creating packets, was modeled after the existing ns-2 UDP agent. The actual packets our agent was sending were UDP packets, however this doesn't impact anything of interest to us since the IP layer is what we are interested in. This agent was responsible for randomizing the marking field before transmission, as would be done to the IP identification field by the OS in a real network node. This agent was also responsible for initializing a few other variables we had added to each IP packet in order to make certain aspects of the simulation easier to deal with. Since these other variables do not directly correspond to anything in our design and are only meant to make implementation in ns-2 easier, we will not discuss them in detail.

The second agent we implemented was a traffic sink corresponding to our first agent. This agent would exist at the destination AS during simulation in order to receive the packet, strip out fields of interest and write them to a log file. After the simulation was finished, the log file generated by the traffic sink agent would be analyzed using a separate Java program we had written.

\subsubsection{Modifying Packets Enroute}

The most important part of our implementation in ns- 2 was the code responsible for marking a packet at every AS that supports our solution. In our design, packet 
marking actions are taken at an AS' ingress and egress points. In ns-2 each AS would be represented by a node object. Hence, we needed to find the lines of code in ns2 which would be executed once a packet arrives at a node in order to process it and forward it to the next node. After going through the ns-2 manual, several other supplementary ns-2 documents, and doing a lot of tracing in the code, the appropriate lines of code were located in the recv function of the classifier.cc file. This function is responsible for making forwarding decisions at a node. We make the necessary modifications to the packet in this function before forwarding it to the next hop, or passing it to the traffic sink agent if we are already at the destination AS.

\subsection{Simulation Setup}

Deployment is a critical issue which we have taken into consideration. We have run tests in environments with full deployment of our solution by all ASes, as well as partial deployment.

Our design was implemented in stages to make code development easier, and gain a better understanding of how the addition of more design complexity at each stage impacts the results. In the first stage we implemented the simple marking approach discussed earlier in Section 3.4. This marking scheme is based only on the AS numbers rather than the links between ASes. Since we are using the simple marking scheme in this stage, we cannot support legacy ASes yet, and hence ran simulations based on full deployment of our solution by all ASes. 
In our second stage of development, we enhanced the marking scheme to support legacy ASes as discussed in Section 3.5. At this stage, we still ran simulations with full deployment of our solution by all ASes so that we could compare the difference in results due to the change in the marking scheme.

For each of these two stages where we had no legacy ASes present (full deployment), we tested our approach using the Inet 3 topology by running 20 separate simulations. For each simulation, one AS among the 5000 ASes was selected randomly as our target AS to lessen the dependence of the results on the location of the chosen AS in the topology. The other 4999 ASes directed traffic towards the target AS. Among these 4999 ASes, 500 were randomly chosen to send spoofed packets, while the rest sent non-spoofed packets. This amounts to 40 simulations in total for these two development stages in order to assess the feasibility of our solution. For all these 40 simulations, the same settings in terms of the marking field length and number of bits per mark were used. A few other simulations were also executed for the first stage in order to evaluate the effect of manipulating variables such as the total number of marks recorded in the marking field, as well as the number of bits allocated to each mark.

In the third and final stage of implementation we used our legacy-supporting marking scheme in various partial deployment scenarios. To test our approach with legacy support, we randomly chose 5 ASes as the target. For each of these we ran 8 simulations where the percentage of legacy ASes present was raised from $10 \%$ in the 
first simulation to $80 \%$ in the last. This amounts to 40 simulations for each scenario with legacy support. We ran four such scenarios with Inet3 topology, and three others with the Brite topology. The specifics of these scenarios are discussed in Section 4.3.1.

\subsubsection{Test Scenarios Executed}

Our initial test scenarios were carried out with a 16-bit marking field. This is because most current IP traceback approaches only use the 16-bit IP identification field. Our tests on the first and second implementation stages which involved full deployment by all the ASes were carried out with the following settings: Inet3 topology, 16bit marking field, 4 marks in total, 4 bits per mark. This corresponds to the 40 simulations previously mentioned. The results for our first implementation stage are presented in Section 5.1.

We ran a few additional test scenarios with our first stage of implementation to evaluate the effects of modifying the number of bits used per AS mark, and the total number of AS marks recorded. When modifying each of these two variables, the other variable was held constant, therefore the size of the marking field was different in each test scenario. The following scenarios were tested with the Inet3 topology and their results are presented in Section 5.2:

- 4 marks in total, 4 bits per mark, 16-bit marking field

- 4 marks in total, 5 bits per mark, 20-bit marking field 
- 4 marks in total, 6 bits per mark, 24-bit marking field

- 5 marks in total, 5 bits per mark, 25-bit marking field

- 6 marks in total, 5 bits per mark, 30-bit marking field

Our second implementation stage was tested with the exact same settings as our first stage. The only difference between the two scenarios was the enhanced marking scheme of the second stage. The corresponding results are presented in Section 5.3.

Our third implementation stage which involved partial deployment situations was tested with the following scenarios:

1. Inet3, 16-bit marking field, 4 marks, 4 bits/mark

2. Inet3, 30-bit marking field, 3 marks, 10 bits/mark

3. Inet3, 31-bit marking field, 4 marks +3 partial bits of the 5 th mark, 7 bits/mark

4. Inet3, 30-bit marking field, 5 marks, 6 bits/mark

5. Inet3, 30-bit marking field, 6 marks, 5 bits/mark

6. Brite, 31-bit marking field, 4 marks +3 partial bits of the 5 th mark, 7 bits $/$ mark

7. Brite, 30-bit marking field, 5 marks, 6 bits/mark

8. Brite, 30-bit marking field, 6 marks, 5 bits $/$ mark 
Scenarios 1 and 3 above are meant to test the effects of moving from a 16-bit marking field to a 31-bit marking field. The corresponding results are presented in Section 6.1 .

The effects of moving from the Inet3 topology to the Brite topology can be studied by comparing the results from scenarios 3 and 6 , which are presented in Section 6.2.

Finally we can study the effects of varying the number of marks and number bits per AS mark while keeping the marking field length pretty much constant. This is achieved by comparing scenarios 2 to 8 , the results of which are presented in Section 6.3.

Our use of a 30/31-bit marking field here corresponds to our discussion in Section 3.3 where we propose a method for using additional bits in the IP header compared to what is currently used by most packet marking proposals. In some of our scenarios above we used a 30 bit marking field instead of 31 bits. The extra one bit adds very little to the accuracy, while requiring many more path signature id reply messages from the sending AS in order to isolate and remove random initialization bits from the path signature.

\subsubsection{Simulation Phases}

The main focus of these simulations was to check the effectiveness of our solution in identifying origin ASes and how incremental deployment affects it. Therefore, while we made considerable modifications in ns-2 to implement our design, we used a simple 
signature distribution method rather than the ones we suggested in Section 3.7.

Each simulation has 3 phases as shown below:

1. The other 4999 ASes send path signature id reply packets to the target AS to populate its database of AS signatures. This phase constitutes our simple signature distribution mechanism.

2. The other 4999 ASes send non-spoofed packets to the target AS to see whether or not the target AS can successfully match the packet signature with the one it is expecting. In the third stage of development with legacy support, there is some boostrapping that occurs when receiving this normal traffic. This is due to the fact that the target AS may have learned multiple path signatures for a legacy AS in phase 1, and it needs to figure out which one, if any, it is actually using to send us traffic.

3. A select number of ASes are selected as attackers (currently using 500 or $10 \%$ of all ASes in the topology) and these send spoofed packets to the target AS to see how well it can trace them back to the potential source AS based on what it has learned in the previous two phases.

\subsection{Analyzing Simulation Results}

As stated earlier the simulation log file generated by the traffic sink agent in ns-2 was analyzed using a separate program written in Java. Java's extensive library of 
built in data structures makes it well suited for parsing the log file, and collecting and computing the necessary statistics.

In order to perform AS-path signature lookups, we took the implementation of a radix tree data structure [45], which is based on a trie data structure, and added new functionality to it. The original functionality primarily consisted of returning all stored entries that start with a given prefix. The functionality we added was so that when we extract the value in the marking field of a packet, we can compare it to all known AS-path signatures and retrieve all matching AS-path signatures of the same or shorter lengths. This directly translates to our LMS and AMS traceback techniques. The functionality we added was essentially the reverse of what was originally supported by the radix tree.

Some of the statistics we collected from each simulation log file include:

- Number of ASes we recovered path signatures for

- Number of legacy ASes we recovered valid path signatures for

- Number of legacy ASes with no valid path signatures

- Number of unique AS signatures

- Number of duplicate AS signatures

- Number of spoofed packet signature match successes

- Avg Number of possible source ASes identified for each spoofed packet 


\section{Chapter 5}

\section{Results and Performance Analysis}

\section{with Full Deployment}

As discussed earlier, our design was implemented in stages to make code development easier, and gain a better understanding of how the addition of more design complexity at each stage impacts the results. In the first stage we implemented the simple marking approach which is based only on the AS numbers rather than the links between ASes. Legacy ASes are not supported at this stage, and hence we ran simulations based on full deployment of our solution by all ASes.

Our second stage of development included an enhanced marking scheme to support legacy ASes. We still ran simulations with full deployment at this stage so that we could compare the difference in results due to the change in the marking scheme alone. 
In this Chapter we will present the results and analysis for the first two stages of development. This includes a few brief tests to assess the impact of increasing the number of bits per AS mark, versus the total number of marks in the packet, on the accuracy of the results.

In Chapter 6 we present the results and analysis for the third and final stage of implementation, where we used our legacy-supporting marking scheme in various partial deployment scenarios.

\subsection{Traceback Using the Simple Marking Scheme}

The results shown in this section are based on the simple marking scheme with a marking field that was 16 bits long and could accommodate four 4-bit AS marks. As shown in table 5.1 when we used the AMS technique to trace a packet, on average it narrowed down the source AS to a list of 87.25 ASes out of the entire AS map. This list successfully contained the true source AS $100 \%$ of the time (by design) in a full deployment scenario. Using only the LMS technique narrowed down the source AS to a list that is on average only 31.1 ASes out of the entire AS map. This list contained the actual source AS in $75.57 \%$ of the cases.

When performing traceback in such a situation where there is a sizeable gap between the success rate of our two traceback techniques, we can first use the LMS technique and send parallel queries to the local IP traceback system of only the very small list of potential source ASes it identifies. In the majority of cases ( $75.57 \%$ here) 


\begin{tabular}{|l|l|l|}
\hline $\begin{array}{l}\text { Traceback } \\
\text { technique }\end{array}$ & $\begin{array}{l}\text { Avg \# of potential } \\
\text { source ASes }\end{array}$ & $\begin{array}{l}\text { \% of correct IP } \\
\text { traceback matches }\end{array}$ \\
\hline LMS & 31.1 & $75.57 \%$ \\
\hline AMS & 87.25 & $100 \%$ (by design) \\
\hline
\end{tabular}

Table 5.1: Traceback results with full deployment (simple marking strategy, 16-bit marking field, 4 marks)

one of those ASes would reply and identify itself as the source. If this fails, then we can try querying the longer list of possible source ASes identified using the AMS technique. Using this combined LMS-AMS approach ensures that we send out as few query messages as necessary.

\subsection{Effect of Number of Bits Per AS Mark and Number of Marks}

A few simulations with varying number of bits per AS mark, and varying number of total marks revealed that allocating more bits to each AS mark significantly increased the accuracy of the results, while increasing the number of total marks beyond 4 , results in minimal gain in the topology we were working with.

For these simulations we chose one representative target AS to test with, while the results in the previous section are an average based on results obtained for 20 target ASes. In tables 5.2 and 5.3, we keep the number of total AS marks in the packet constant at 4 , but increase the number of bits used for each AS mark. 


\begin{tabular}{|l|l|l|l|}
\hline $\begin{array}{l}\text { Traceback } \\
\text { technique }\end{array}$ & $\begin{array}{l}\text { Avg \# of poten- } \\
\text { tial source ASes } \\
\left(\begin{array}{l}\mathbf{4} \text { bits per AS } \\
\text { mark })\end{array}\right.\end{array}$ & $\begin{array}{l}\text { Avg of poten- } \\
\text { tial source ASes } \\
\left(\begin{array}{l}5 \text { bits per AS } \\
\text { mark })\end{array}\right.\end{array}$ & $\begin{array}{l}\text { Avg of poten- } \\
\text { tial source ASes } \\
7 \text { bits per AS } \\
\text { mark })\end{array}$ \\
\hline LMS & 15 & 11 & 5 \\
\hline AMS & 92 & 58 & 20 \\
\hline
\end{tabular}

Table 5.2: Effect of increasing the size of each AS mark on the list of potential source ASes (simple marking strategy, 4 marks in total)

\begin{tabular}{|c|c|c|c|}
\hline $\begin{array}{l}\text { Traceback } \\
\text { technique }\end{array}$ & 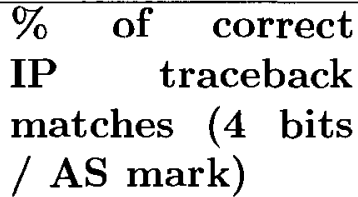 & $\begin{array}{l}\text { \% of correct } \\
\text { IP traceback } \\
\text { matches }(5 \text { bits } \\
\text { / AS mark })\end{array}$ & 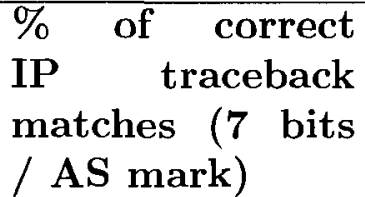 \\
\hline LMS & $64.03 \%$ & $80.99 \%$ & $95.7 \%$ \\
\hline
\end{tabular}

Table 5.3: Effect of increasing the size of each AS mark on the traceback success rate (simple marking strategy, 4 marks in total)

We also ran another set of tests where the size of each AS mark was kept at 5 bits long, but the total number of marks in the packet was increased from 4 , to 5 , and then 6 . Increasing the number of marks made no difference in the number of potential source ASes identified. It had only a very minimal effect on the success rate of containing the actual source AS in the identified list.

\subsection{Effect of Marking Strategy}

The results shown in this section were obtained using our stage 2 implementation, which is based on the legacy-supporting marking scheme. Although this marking strategy supports legacy ASes, the results here are based on full deployment so that 
we can compare the impact of this marking strategy with the simple marking strategy.

As is evident by comparing the data shown in tables 5.1 and 5.4 , there is little to no significant difference in the accuracy of our results between the two marking strategies.

\begin{tabular}{|l|l|l|}
\hline $\begin{array}{l}\text { Traceback } \\
\text { technique }\end{array}$ & $\begin{array}{l}\text { Avg \# of potential } \\
\text { source ASes }\end{array}$ & $\begin{array}{l}\text { \% of correct IP } \\
\text { traceback matches }\end{array}$ \\
\hline LMS & 31.5 & $75.43 \%$ \\
\hline AMS & 88.55 & $100 \%$ (by design) \\
\hline
\end{tabular}

Table 5.4: Traceback results with full deployment (legacy-supporting marking strategy, 16-bit marking field, 4 marks)

The was no difference encountered between the two marking strategies in terms of the number of path signature id reply packets required in order to form valid signatures for the source ASes. We needed a minimum of 6 path signature id reply packets with both marking strategies. 


\section{Chapter 6}

\section{Results and Performance Analysis}

\section{with Partial Deployment}

In this Chapter we present the results and analysis for the third and final stage of implementation, where we used our legacy-supporting marking scheme in various partial deployment scenarios.

There is now a possibility that using the AMS traceback technique, our list of potential sources will not contain the true origin because it might be a legacy AS for which we haven't been able to obtain a path signature. It is most likely that even if it is not possible to identify the source AS, we can still identify the most upstream traceable AS. This is not taken into consideration here, but we will explore this in our future work.

We can successfully gather path signatures for a significant percentage of legacy 
ASes, even when there are many more legacy ASes than ones that support our solution. In the Inet3 topology, with a low deployment of $20 \%$ to $40 \%$ of ASes supporting our solution, we can recover signatures for $43 \%$ to $53 \%$ of the legacy ASes present. With the Brite topology these numbers are somewhat lower, however increasing the deployment rate results in a greater gain in the number of legacy ASes we can recover signatures for when compared to the Inet3 topology.

\subsection{Marking Field Length}

In this section we examine the effects of moving from a 16-bit marking field to a 31-bit marking field. For these simulations we used the Inet3 topology. We chose to record 4 marks in the packet based on the fact that this topology has a mean distance of 3.54. When using a 16-bit marking field we set each AS mark to be 4 bits in length, and 7 bits in length when using a 31-bit marking field. Using 7-bit marks in a 31-bit wide field leaves 3 extra bits. These bits are useful in cases where the path length is longer than 4 ASes. For example if the path is 5 ASes long, the packet header would contain full marks for the last 4 ASes as well as 3 marking bits of the very first AS in the path.

In table 6.1 we present how many ASes on average the potential source AS can be narrowed down to out of all possible 4999 ASes (besides the target AS) in the topology. As is evident, the greater accuracy conferred by the 31-bit marking field allows quite a few more potential source ASes to be ruled out. 


\begin{tabular}{|l|l|l|}
\hline $\begin{array}{l}\text { Traceback } \\
\text { technique }\end{array}$ & $\begin{array}{l}\text { Avg \# of potential } \\
\text { source ASes (16-bit } \\
\text { field) }\end{array}$ & $\begin{array}{l}\text { Avg \# of potential } \\
\text { source ASes (31-bit } \\
\text { marking field) }\end{array}$ \\
\hline LMS & 14.6 & 5.6 \\
\hline AMS & 97.3 & 21.5 \\
\hline
\end{tabular}

Table 6.1: Traceback results with partial deployment (Inet3 topology with 5000 ASes, 16-bit vs 31-bit marking field, 4 marks)

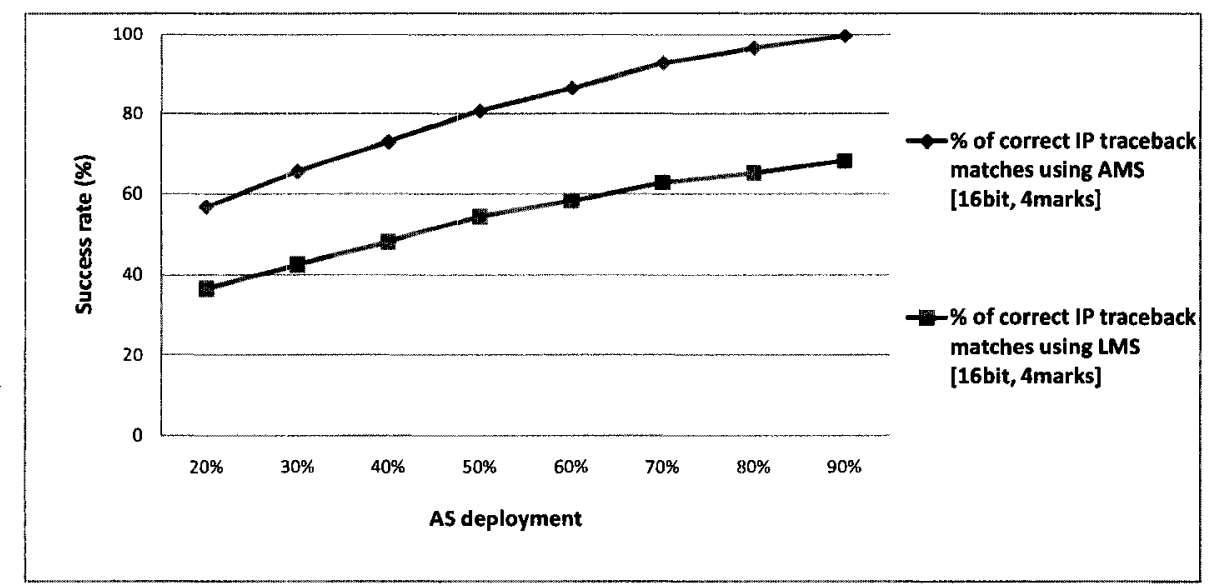

Figure 6.1: Comparison of traceback success rates using AMS and LMS methods (Inet3 topology, 16-bit marking field, 4 marks)

In Fig. 6.1 we illustrate how the rate of successfully containing the true origin AS among the list of potential ASes identified using our two traceback methods varies with the deployment rate. This figure corresponds to a 16-bit marking field. As expected, this rate goes down as the number of legacy ASes present rises. However, this rate is still pretty good even in the extreme case when only $20 \%$ of the ASes support our solution. In this case the LMS approach contains the true origin AS among 14.4 possible source ASes $37 \%$ of the time, while the AMS approach contains the true origin AS among 90 potential source ASes $57 \%$ of the time. 
In this situation there is a sizeable gap between the success rate of our two traceback techniques. As was discussed in Section 5.1, we can use the combined LMS-AMS approach to ensure that we send out as few query messages as possible.

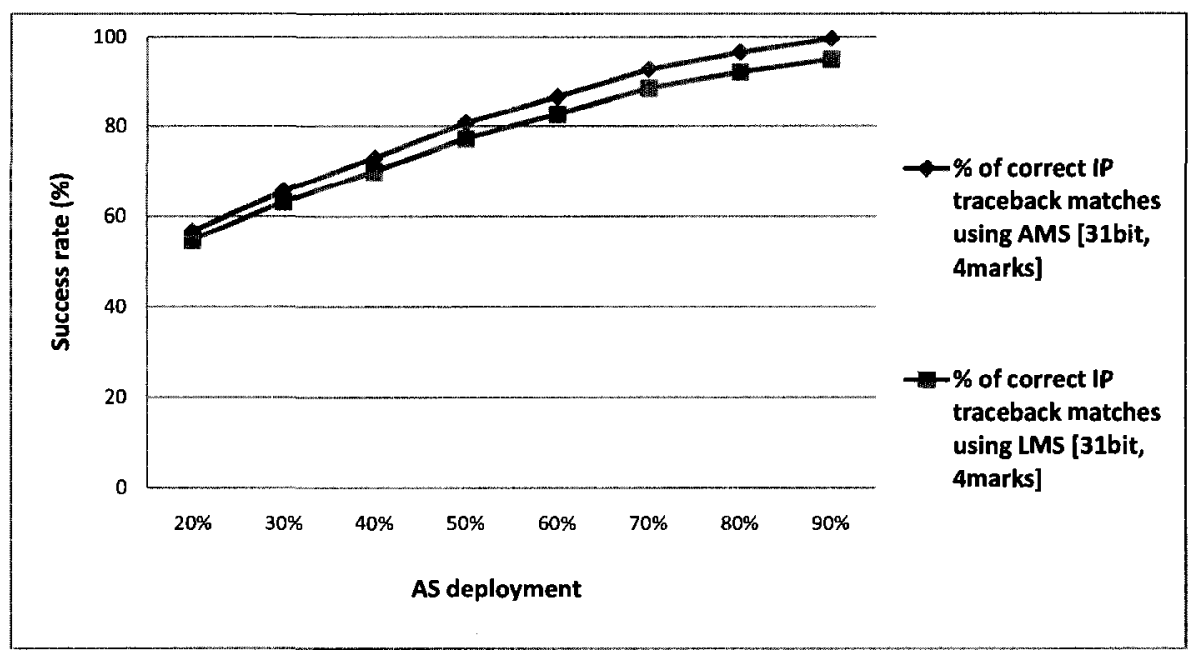

Figure 6.2: Comparison of traceback success rates using AMS and LMS methods (Inet3 topology, 31-bit marking field, 4 marks)

The results are quite different however when we use a 31-bit marking field as shown in Fig. 6.2. The success rate of the LMS approach is much higher than when using a 16-bit marking field. The LMS success rate is actually almost the same as that of the AMS method. With regards to the combined LMS-AMS traceback approach we previously mentioned, the results indicate that when the quality of the path signatures is as high as it is in this case there would be little need to go beyond the first tier which involves querying the list of potential source ASes identified with the LMS technique. The combined LMS-AMS traceback querying approach is best used if we are restricted to use a short marking field, such as 16 bits, and when the 
path signatures are not nearly as unique as they are in this case.

\subsection{Effect of Topology}

As mentioned in Section 4.1.1, besides the Inet3 generated topology we also used a topology generated with Brite which gave us a longer average AS-path length. This longer path length should be better representative of the current Internet average AS-path length.

We have evaluated the difference in performance of our scheme between the two topologies when using a 31-bit marking field. Based on the data in table 6.2, when using the Brite topology the potential source AS can be narrowed down to fewer ASes. However, the traceback success rate is somewhat lower with the Brite topology as illustrated in figures 6.3 and 6.4 .

\begin{tabular}{|l|l|l|}
\hline $\begin{array}{l}\text { Traceback } \\
\text { technique }\end{array}$ & $\begin{array}{l}\text { Avg \# of potential } \\
\text { source ASes (Inet3) }\end{array}$ & $\begin{array}{l}\text { Avg \# of potential } \\
\text { source ASes (Brite) }\end{array}$ \\
\hline LMS & 5.6 & 3 \\
\hline AMS & 21.5 & 13.4 \\
\hline
\end{tabular}

Table 6.2: Comparing traceback results between the Inet3 and Brite topologies (31-bit marking field, 4 marks) 
Results and Performance Analysis with Partial Deployment

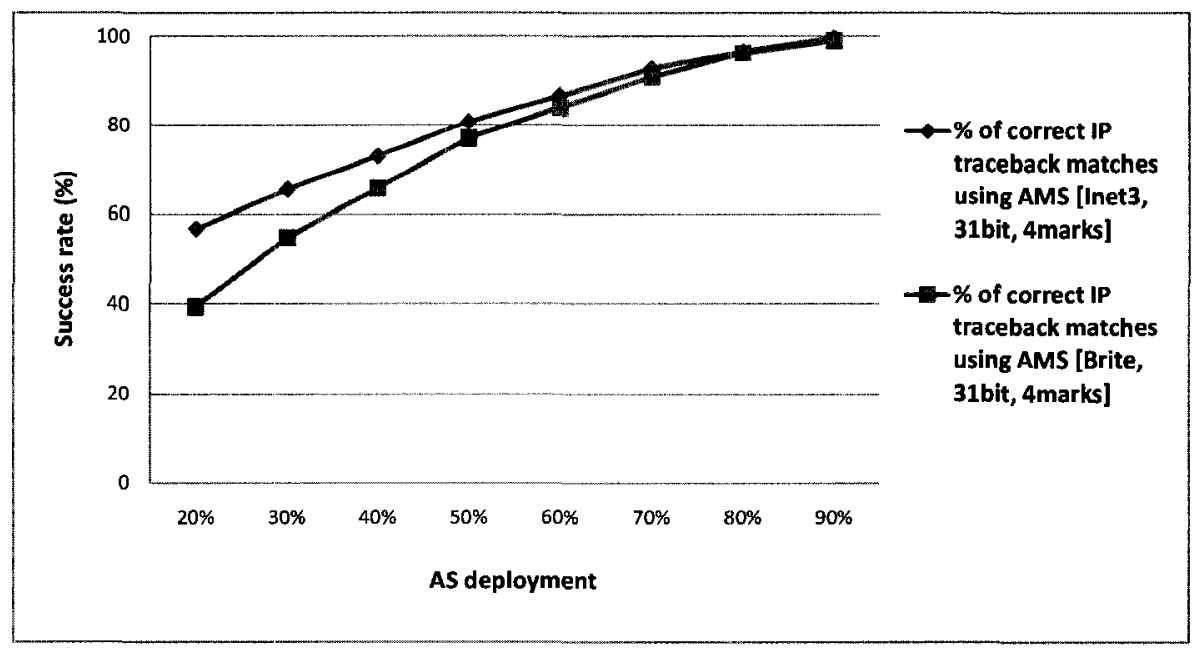

Figure 6.3: Comparing AMS traceback success rates between Inet3 and Brite topologies (4 marks)

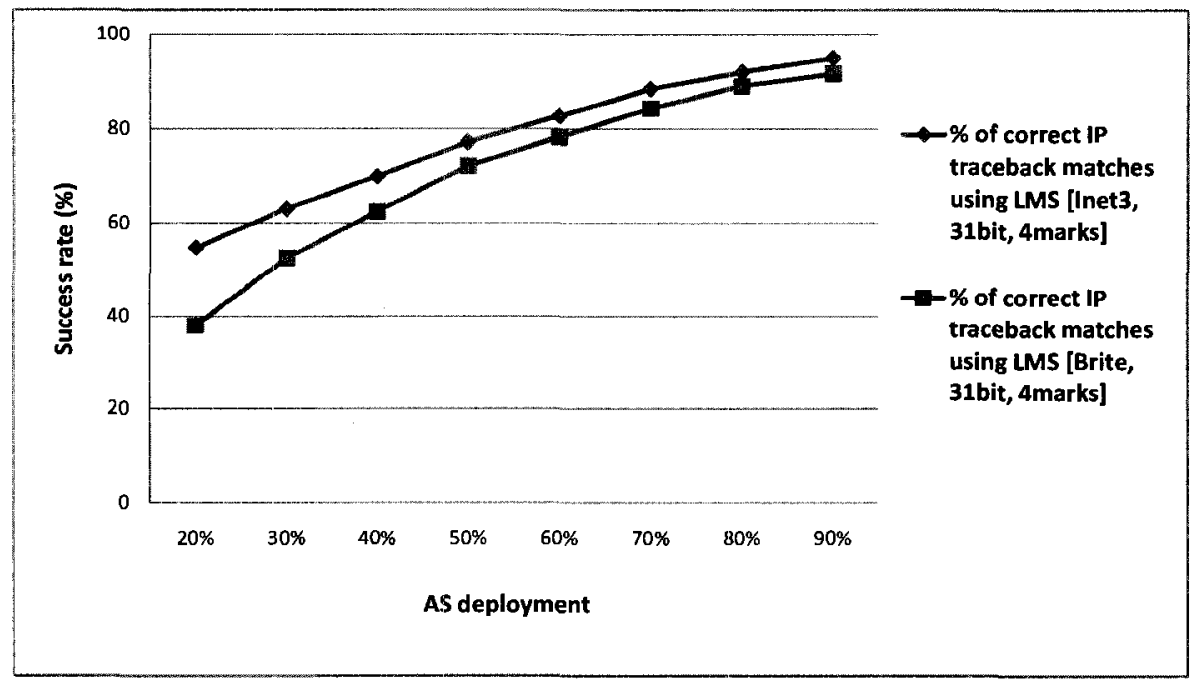

Figure 6.4: Comparing LMS traceback success rates between Inet3 and Brite topologies (4 marks) 


\subsection{Varying the Number of Marks Recorded}

In this section we evaluate the effects of varying the number of total marks stored in the packet using both the Brite and Inet3 topologies. Given a fixed-size marking field, increasing the number of marks means decreasing the number of bits per mark.

In tables 6.3 and 6.4 we show how many ASes on average the potential source AS can be narrowed down to out of all possible 4999 ASes in each of our two topologies.

\begin{tabular}{|l|l|l|l|}
\hline $\begin{array}{l}\text { Traceback } \\
\text { technique }\end{array}$ & $\begin{array}{l}\text { Avg \# of poten- } \\
\text { tial source ASes } \\
\text { (4 marks) }\end{array}$ & $\begin{array}{l}\text { Avg \# of poten- } \\
\text { tial source ASes } \\
\text { (5 marks) }\end{array}$ & $\begin{array}{l}\text { Avg \# of poten- } \\
\text { tial source ASes } \\
\text { (6 marks) }\end{array}$ \\
\hline LMS & 3 & 2.5 & 3.3 \\
\hline AMS & 13.4 & 15 & 27 \\
\hline
\end{tabular}

Table 6.3: Traceback results with partial deployment (Brite topology with 5000 ASes, 4 to 6 marks)

\begin{tabular}{|c|c|c|c|c|}
\hline $\begin{array}{l}\text { Traceback } \\
\text { technique }\end{array}$ & $\begin{array}{l}\text { Avg \# of } \\
\text { potential } \\
\text { source ASes } \\
\text { (3 marks) }\end{array}$ & $\begin{array}{l}\text { Avg \# of } \\
\text { potential } \\
\text { source ASes } \\
(4 \text { marks })\end{array}$ & $\begin{array}{l}\text { Avg \# of } \\
\text { potential } \\
\text { source ASes } \\
\text { (5 marks) }\end{array}$ & $\begin{array}{l}\text { Avg \# of } \\
\text { potential } \\
\text { source ASes } \\
(6 \text { marks })\end{array}$ \\
\hline LMS & 96.3 & 5.6 & 7.2 & 11.8 \\
\hline $\mathrm{AMS}$ & 141.10 & 21.4 & 29.0 & 54.2 \\
\hline
\end{tabular}

Table 6.4: Traceback results with partial deployment (Inet3 topology with 5000 ASes, 3 to 6 marks)

The data in table 6.3 indicates that choosing to allow 5 marks in the packet header gives the most accurate results with the Brite topology. This number of marks results in the lowest number of potential source ASes when using the LMS technique. It should be noted that this choice most closely matches the mean distance of the Brite topology which was found to be 4.76 . When using the AMS technique, the number 
of potential source ASes identified using 5 marks is only slightly higher than when using 4 marks. Our best choice still remains 5 marks however, because in this case LMS results are more important than AMS results. The reason for this will become more clear later on in this section when we compare the success rate of LMS versus AMS.

The data in table 6.4 indicates that choosing to place 4 marks in the packet header is the best option when using the Inet3 topology. This choice results in the fewest potential source ASes identified using either one of our two traceback techniques. The mean distance of the Inet3 topology was found to be 3.54. Hence, once again our choice most closely matches the mean distance of the topology being used. This set of tests included a case with 3 marks whereas the tests with the Brite topology did not include this number of marks. The reason for this is that it was desired to have at least one case with fewer marks than the mean distance of the topology.

Now that we have looked at choosing the ideal number of marks in the packet based on the number of potential ASes identified, let us look at another set of results and see if we arrive at the same conclusions. Fig. 6.5 illustrates, using the Brite topology, how the rate of successfully identifying the true origin AS among the list of potential ASes obtained using the LMS approach varies with the deployment rate. The success rate using only the AMS technique is by design the same among the different simulations run on the same topology. Based on these results, choosing to allow 5 marks in the packet header gives the most accurate results. As is evident, our 
two sets of results for the lowest number of potential source ASes identified (table 6.3), and the highest success rate in identifying the true origin AS (Fig. 6.5) indicate that choosing the number of marks in the packet to be closest to the mean distance of the topology gives the best results.

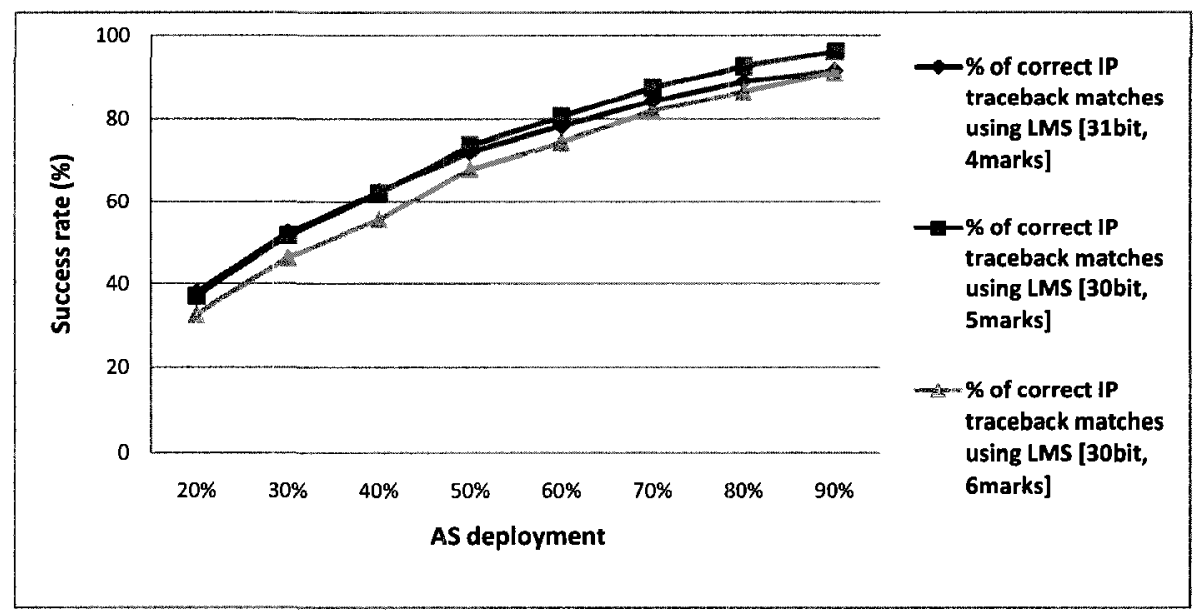

Figure 6.5: LMS traceback success rates comparison (Brite topology, 4 to 6 marks)

Fig. 6.6 illustrates a similar idea using the Inet3 topology. The main difference here is that the highest LMS success rate is achieved with 3 marks as opposed to 4 marks which is closest to the topology's mean distance of 3.54 . However, this high success rate should be taken with a grain of salt because it is achieved through identifying a very high number of potential source ASes compared to cases with 4 , 5, or 6 marks. Because of this inefficiency in LMS results, choosing to place 3 marks in the packet is not an ideal choice. Taking into account the two results sets from table 6.4 and Fig. 6.6, the best option is to choose 4 marks in the packet, which closely matches the topology's mean distance. This would give us the lower number 
of potential source ASes identified, with a very high success rate.

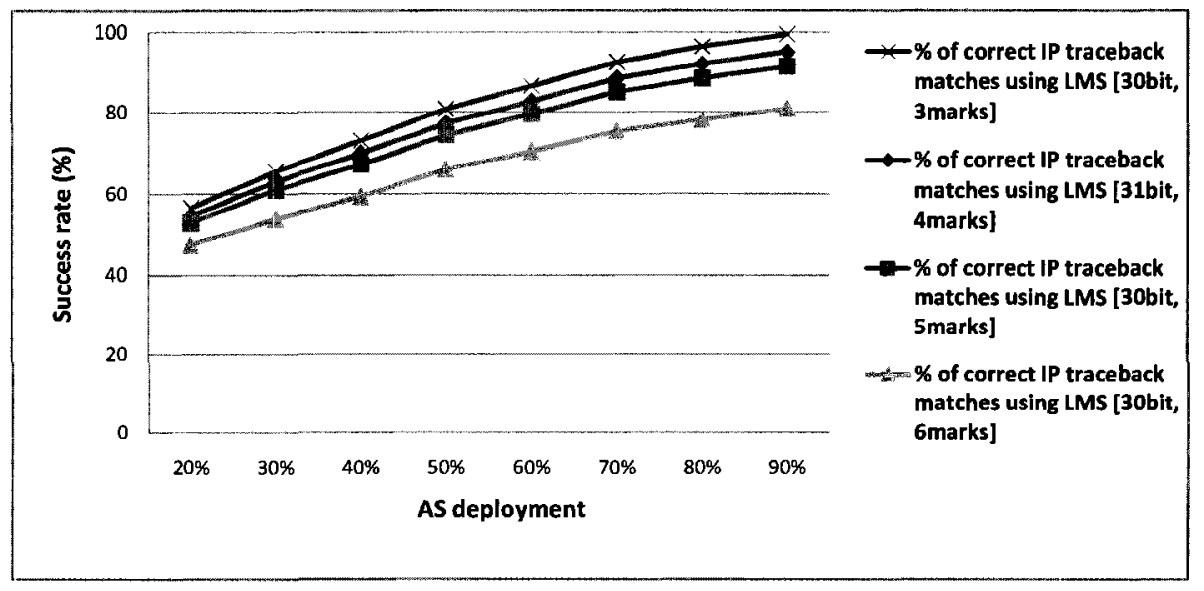

Figure 6.6: LMS traceback success rates comparison (Inet3 topology, 3 to 6 marks)

In section 6.1, for the case involving the Inet3 topology with a 31-bit marking field (Fig. 6.2), we concluded that the success rate of the LMS technique is very close to that of the AMS technique. Therefore, there is negligible gain in using the combined LMS-AMS traceback approach in such a situation. We have a similar situation with the Brite topology as illustrated in Fig. 6.7. Since it is almost completely sufficient to use just the LMS technique in this situation, we can place our emphasis on the LMS results when deciding what metrics to choose such as the most appropriate number of marks allowed in the packet header. 


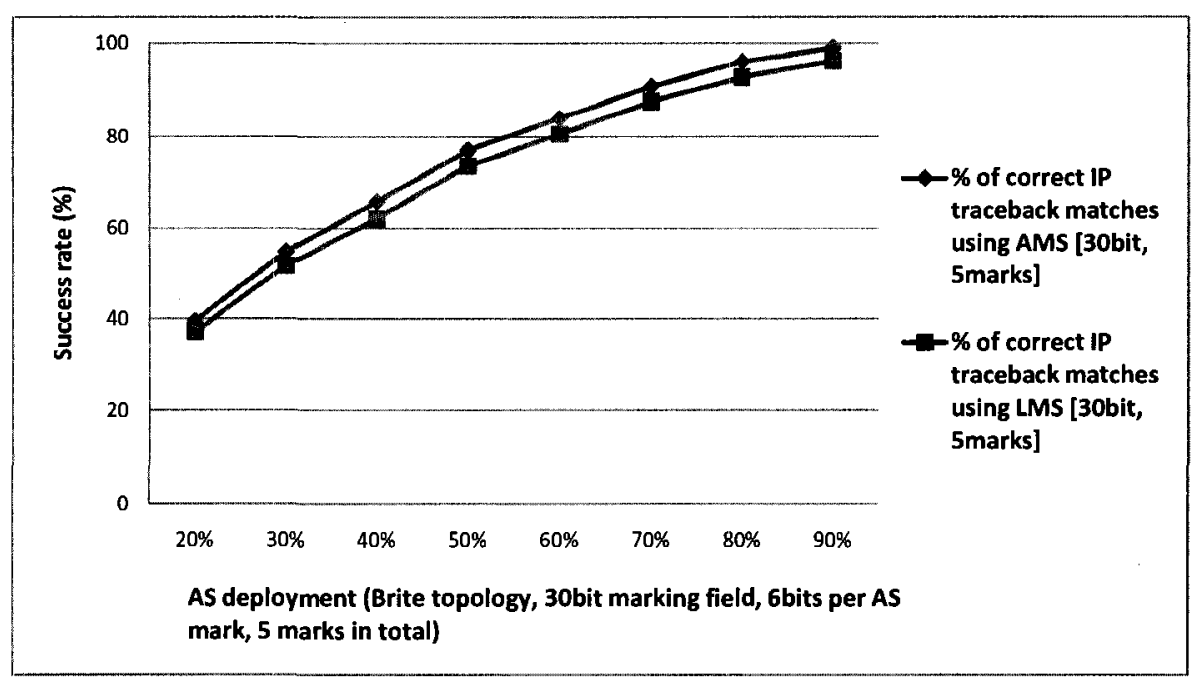

Figure 6.7: Comparison of traceback success rates using AMS and LMS methods (Brite topology, 30-bit marking field, 5 marks)

\subsection{Effect of Marking Field on Learning Path Sig-}

\section{natures}

In this section we analyze the number of path signature id reply packets required in order to form valid path signatures in our simulations. What we expect is that as the number of bits used per AS mark increases, the number of path signature id reply packets required decreases. We expect this because with longer AS marks, it would be much more difficult for any random initial data in the marking field to exactly match an AS mark across multiple path signature id reply packets.

Inet3 Topology: 
- 31-bit marking field, 7bits/AS, 4 marks $\Longrightarrow 4$ packets

- 30-bit marking field, 6 bits/AS, 5 marks $\Longrightarrow 5$ packets

- 30-bit marking field, 5bits/AS, 6 marks $\Longrightarrow 5$ packets

- 16-bit marking field, 4bits/AS, 4 marks $\Longrightarrow 6$ packets

- 31-bit marking field, 7bits/AS, 4 marks +3 partial bits $\Longrightarrow 7$ packets

Brite Topology:

- 31-bit marking field, 7bits/AS, 4 marks $\Longrightarrow 4$ packets

- 30-bit marking field, 6bits/AS, 5 marks $\Longrightarrow 5$ packets

- 30-bit marking field, 5bits/AS, 6 marks $\Longrightarrow 5$ packets

- 31-bit marking field, 7bits/AS, 4 marks +3 partial bits $\Longrightarrow 6$ packets

Given the data here, we can confirm our expectation that the more bits used per AS mark, the fewer path signature id reply packets required. The higher number of packets needed when using 7 bits per AS mark to record 4 full marks, along with 3 partial bits of the 5 th mark is due to those partial bits. When the same set of simulations results are analyzed without taking those 3 partial bits into account, fewer path signature id reply packets are required. 


\section{Chapter 7}

\section{Conclusions and Future Work}

\subsection{Conclusions}

The hierarchical solution we have developed provides a means carrying out IP traceback at the AS-level, while allowing for incremental deployment. It can also enable filtering incoming attack packets at the network edge, and detecting spoofed IP packets. Our solution can work on both live incoming traffic, as well as on previously captured packets.

In our discussions, we suggested a means of using more fragmentation related bits in the IPv4 header than what is typically used in current proposed IP traceback schemes. We proposed two different methods for carrying out IP traceback in our solution. These were the All Matching Signatures and Longest Matching Signature techniques. 
We carried out a number of simulation experiments in scenarios with both full deployment, and partial deployment of our solution. We used two separate topologies in our tests. We tested the effects of varying the sizes of the marking field, varying the number of total AS marks, as well as using different sizes for each AS mark.

Our simulation results showed that we can recover signatures for a large number of legacy ASes and that we can perform traceback with good accuracy in the presence of legacy ASes. We concluded that the most appropriate choice for the number of marks to allocate in a packet is that which corresponds to the average AS path length of the topology. We observed that increasing the size of each AS mark has a far more significant effect on the accuracy of the results compared to increasing the total number of marks.

When the length of the marking field is small, 16 bits for example, we observed a sizeable gap between the success rates of our two traceback techniques. We concluded that the best approach in such a situation is to use a combined LMS-AMS technique to ensure that we send out as few query messages to potential source ASes as possible. With longer marking fields, in the range of 31 bits, we noticed the gap between the success rates of the two traceback techniques becomes much narrower. Thus, we concluded that in such situations using only the LMS technique would likely be sufficient.

We believe the numerous strong points mentioned should make our solution a good candidate for future adoption, or serve as the basis for future work in this area. 


\subsection{Future Research}

In this thesis we presented results based on simulations. Our research can benefit from analytical evaluation as well. Particularly in terms of determining the minimum number of path signature id reply packets that should be sent so that the recipient can distinguish valid marking bits from initial random data in the marking field.

Our research was primarily centered around IP traceback. Hence, we used a very simple signature distribution mechanism for our simulations. We did not test the candidate signature distribution algorithms we proposed in Section 3.7. This is an area which can be the focus of further research and fine-tuning.

Within the context of signature distribution, we mentioned a number of variables, the fine-tuning of which can be the subject of future research. These variables were as follows:

- $t$, which is the minimum time delay between sending path signature id reply packets to the same destination AS

- $k$ time units, at every countdown of which the dedicated server will send out path signature id reply messages to an AS 


\section{Appendix A}

\section{NS-2 Modification Summary}

In table A.1 we list all the ns-2 files modified in order to implement our solution. We also provide a brief description of why each file was modified.

\begin{tabular}{|l|l|l|}
\hline Filename & Location & Reason for Modification \\
\hline astackpisig.cc & /apps & $\begin{array}{l}\text { Newly added agent to construct and initial- } \\
\text { ize our simulation packets. Functionalities } \\
\text { such as randomizing the contents of the IP } \\
\text { identification field are performed here. }\end{array}$ \\
\hline astackpisig.h & /apps & Newly added header file for astackpisig.cc \\
\hline astackpisigsink.cc & /apps & $\begin{array}{l}\text { Newly added traffic sink agent to consume } \\
\text { our simulation packets and write fields of in- } \\
\text { terest to a log file for later analysis. This } \\
\text { would include information on the type of } \\
\text { packet, such as a spoofed packet, normal } \\
\text { packet, or one meant to be used for learn- } \\
\text { ing path signatures. The marking field of } \\
\text { the packet would also be written to the log } \\
\text { file. The source AS number, and whether or } \\
\text { not it is a legacy AS would also be recorded. }\end{array}$ \\
\hline astackpisigsink.h & /apps & $\begin{array}{l}\text { Newly added header file for astack- } \\
\text { pisigsink.cc }\end{array}$ \\
\hline
\end{tabular}




\begin{tabular}{|c|c|c|}
\hline classifier.cc & / classifier & $\begin{array}{l}\text { Added a considerable amount of code to recv } \\
\text { function to perform the actual packet mark- } \\
\text { ing according to our solution design. The } \\
\text { Classifier class is the most important class } \\
\text { in ns } 2 \text { as far as our solution is concerned. } \\
\text { If we were to approximate how ns } 2 \text { simula- } \\
\text { tions run as compared to the operations in a } \\
\text { real physical network, the Classifier class per- } \\
\text { forms forwarding functionalities in a router. } \\
\text { The functionalities of the ingress and egress } \\
\text { routers discussed in our solution, and gener- } \\
\text { ating signatures on behalf of legacy AS neigh- } \\
\text { bours are all done in this class. }\end{array}$ \\
\hline classifier.h & /classifier & $\begin{array}{l}\text { Added additional variable to keep track of } \\
\text { which node this classifier object is associated } \\
\text { with }\end{array}$ \\
\hline ip.h & /common & $\begin{array}{l}\text { Added several new fields to each IP packet } \\
\text { such as the actual marking field, and others } \\
\text { to help make implementation easier by keep- } \\
\text { ing track of the simulation stage, or to record } \\
\text { the full route taken by the packet thus far, } \\
\text { etc. }\end{array}$ \\
\hline node.cc & /common & $\begin{array}{l}\text { Added code to keep track of whether or not } \\
\text { this node supports our solution }\end{array}$ \\
\hline node.h & /common & $\begin{array}{l}\text { Added code to keep track of whether or not } \\
\text { this node supports our solution }\end{array}$ \\
\hline ns-default.tcl & $/ \mathrm{tcl} / \mathrm{lib}$ & $\begin{array}{l}\text { Modifications needed to support newly } \\
\text { added agents. These had to do with the de- } \\
\text { fault values some of the variables in a new in- } \\
\text { stance of our agent would be initialized with } \\
\text { upon creation. }\end{array}$ \\
\hline ns-node.tcl & $/ \mathrm{tcl} / \mathrm{lib}$ & $\begin{array}{l}\text { Modified so that each node keeps track of } \\
\text { its direct neighbours. Useful in legacy situa- } \\
\text { tion where a solution-supporting node needs } \\
\text { to generate signatures on behalf of its neigh- } \\
\text { bouring legacy ASes. }\end{array}$ \\
\hline
\end{tabular}




\begin{tabular}{|l|l|l|}
\hline & /routing & $\begin{array}{l}\text { Added code to make it much easier to tell } \\
\text { which node a classifier object belonged to. } \\
\text { When first working with ns2 Classifier code, } \\
\text { a big difficulty we had was that there was no } \\
\text { easy way of telling which simulation node the } \\
\text { Classifier code was executing on behalf of. }\end{array}$ \\
\hline simulator.h & $/$ common & $\begin{array}{l}\text { Initialize random number generator when the } \\
\text { simulation starts }\end{array}$ \\
\hline Makefile & $/$ & Include new agent files for compilation \\
\hline
\end{tabular}

Table A.1: List of all ns-2 files modified to implement our design 


\section{Bibliography}

[1] IANA AS Registry. [Online]. Available: http://www.iana.org/assignments/ as-numbers/, (Jan. 2009).

[2] Internet Assigned Numbers Authority. [Online]. Available: http://www.iana. org, (Jan. 2009).

[3] RIPE NCC - RIS Statistics Report. [Online]. Available: http://www.ris.ripe. net/weekly-report/, (Jan. 2009).

[4] Team Cymru - IP to ASN Mapping. [Online]. Available: http://www. team-cymru.org/Services/ip-to-asn.html, (Jan. 2009).

[5] Team Cymru Internet Monitor - BGP Average AS-Path Length. [Online]. Available: http://www.cymru.com/BGP/avg_aspath_len.html, (Jan. 2009).

[6] The CIDR Report. [Online]. Available: http://www.potaroo.net/cgi-bin/ as-report?as $=,($ Jan. 2009). 
[7] The Network Simulator - ns-2. [Online]. Available: http://www.isi.edu/ nsnam/ns/, (Jan. 2009).

[8] IPv6 Extension Headers Review and Considerations, October 2006. [Online]. Available: http://www.cisco.com/en/US/technologies/tk648/tk872/ technologies_white_paper0900aecd8054d37d.html, (Jan. 2009).

[9] TCP Intercept, 2007. Cisco IOS Security Configuration Guide, Release 12.2. [Online]. Available: http://www.cisco.com/en/US/docs/ios/12_2/security/ configuration/guide/scfdenl.html, (Jan. 2009).

[10] Unicast Reverse Path Forwarding, 2007. Cisco IOS Security Configuration Guide, Release 12.2. [Online]. Available: http://www.cisco.com/en/US/docs/ ios/12_2/security/configuration/guide/scfrpf.html, (Jan. 2009).

[11] The Linux Kernel Archives - Version 2.6.26, 2008. [Online]. Available: http: //www.kernel.org/pub/linux/kernel/v2.6/, (Jan. 2009).

[12] E. Albright and X. Dang. An Implementation of IP Traceback in IPv6 Using Probabilistic Packet Marking. PhD thesis, The University of Akron, 2005.

[13] S.O. Amin, M.S. Kang, and C.S. Hong. A Lightweight IP Traceback Mechanism on IPv6, volume 4097 of Lecture Notes in Computer Science. Springer Berlin, 2006. 
[14] A. Belenky and N. Ansari. On IP Traceback. Communications Magazine, IEEE, 41(7):142-153, 2003.

[15] A. Belenky and N. Ansari. Tracing Multiple Attackers with Deterministic Packet Marking (DPM). Communications, Computers and signal Processing, 2003. PACRIM. 2003 IEEE Pacific Rim Conference on, 1:49-52, Aug. 2003.

[16] S.M. Bellovin, M. Leech, and T. Taylor. ICMP Traceback Messages, March 2000. [Online]. Available: http://lasr.cs.ucla.edu/save/rfc/ draft-bellovin-itrace-00.txt, (Jan. 2009).

[17] Burton H. Bloom. Space/Time Trade-offs in Hash Coding with Allowable Errors. Commun. ACM, 13(7):422-426, 1970.

[18] A. Bremler-Barr and H. Levy. Spoofing Prevention Method. In INFOCOM 2005. 24th Annual Joint Conference of the IEEE Computer and Communications Societies. Proceedings of the IEEE, pages 536-547, Mar. 2005.

[19] H. Burch and B. Cheswick. Tracing Anonymous Packets to Their Approximate Source. In LISA '00: Proceedings of the 14th USENIX conference on System administration, pages 319-328, Berkeley, CA, USA, 2000. USENIX Association.

[20] K. Butler and W. Aiello. Optimizing BGP security by exploiting path stability. In Proceedings of the 13th ACM conference on Computer and communications security, pages 298-310. ACM Press New York, NY, USA, 2006. 
[21] André O. Castelucio, Ronaldo M. Salles, and Artur Ziviani. An AS-level IP Traceback System. In CoNEXT '07: Proceedings of the 2007 ACM CoNEXT conference, pages 1-2, New York, NY, USA, 2007. ACM.

[22] A. Dabir and A. Matrawy. Design and Analysis of a Hierarchical IP Traceback System. To be published in IEEE International Conference on Communications (ICC'09), June 2009.

[23] A. Dabir and A. Matrawy. Packet Tracing and Detection of Source-Spoofing Across Multiple Autonomous Systems. Manuscript submitted to a journal for review.

[24] E. Decker, P. Langille, A. Rijsinghani, and K. McCloghrie. Definitions of Managed Objects for Bridges. Internet Request for Comments RFC 1493, 1993.

[25] S. Deering and R. Hinden. Internet Protocol, Version 6 (IPv6) Specification. Internet Request for Comments RFC 2460, 1998.

[26] P. Ferguson and D. Senie. Network Ingress Filtering: Defeating Denial of Service Attacks which employ IP Source Address Spoofing. Internet Request for Comments RFC 2827, 2000.

[27] Z. Gao and N. Ansari. Tracing Cyber Attacks from the Practical Perspective. Communications Magazine, IEEE, 43(5):123-131, 2005. 
[28] C. Gong, T. Le, T. Korkmaz, and K. Sarac. Single Packet IP Traceback in ASlevel Partial Deployment Scenario. In Global Telecommunications Conference, 2005. GLOBECOM'05. IEEE, pages 1817-1821, Nov. 2005.

[29] G. Goodell, W. Aiello, T. Griffin, J. Ioannidis, P. McDaniel, and A. Rubin. Working Around BGP: An Incremental Approach to Improving Security and Accuracy of Interdomain Routing. In In The 10th Annual Network and Distributed System Security Symposium (NDSS03), 2003.

[30] I. Hamadeh and G. Kesidis. A Taxonomy of Internet Traceback. International Journal of Security and Networks, 1(1):54-61, 2006.

[31] J. Hawkinson and T. Bates. Guidelines for creation, selection, and registration of an autonomous system (AS). Internet Request for Comments RFC 1930, 1996.

[32] H. Hazeyama, M. Oe, and Y. Kadobayashi. A Layer-2 Extension to Hash-Based IP Traceback. IEICE Transactions on Information and Systems, 86(11):2325$2333,2003$.

[33] S. Kent, C. Lynn, and K. Seo. Secure Border Gateway Protocol (Secure-BGP). IEEE Journal on Selected Areas in Communications, 18(4):582-592, 2000.

[34] T. Killalea. Recommended internet service provider security services and procedures. Internet Request for Comments RFC 3013, 2000. 
[35] Y. Kim, J.Y. Jo, and FL Merat. Defeating Distributed Denial-of-Service Attack with Deterministic Bit Marking. In Global Telecommunications Conference, 2003. GLOBECOM'03. IEEE, pages 1363-1367, Dec. 2003.

[36] Craig Labovitz. 2008 Worldwide Infrastructure Security Report, November 2008. [Online]. Available: http://asert.arbornetworks.com/2008/11/ 2008-worldwide-infrastructure-security-report/, (Jan. 2009).

[37] Rafael P. Laufer, Pedro B. Velloso, Daniel de O. Cunha, Igor M. Moraes, Marco D. D. Bicudo, Marcelo D. D. Moreira, and Otto Carlos M. B. Duarte. Towards Stateless Single-Packet IP Traceback. In $L C N$ '07: Proceedings of the 32nd IEEE Conference on Local Computer Networks (LCN 2007), pages 548-555, Washington, DC, USA, 2007. IEEE Computer Society.

[38] D. Magoni. nem: A Software for Network Topology Analysis and Modeling. In Proceedings of the 10th IEEE Symposium on Modeling, Analysis and Simulation of Computer \& Telecomm. Systems (MASCOTS02), pages 364-371.

[39] A.M. Mankin, D.C.L.W. Wu, and S.F.L. Zhang. On Design and Evaluation of "Intention-Driven" ICMP Traceback. In Computer Communications and Networks, 2001. Proceedings. Tenth International Conference on, pages 159-165, 2001.

[40] A. Medina, A. Lakhina, I. Matta, and J. Byers. BRITE: An Approach to Universal Topology Generation. In Modeling, Analysis and Simulation of Computer and 
Telecommunication Systems, 2001. Proceedings. Ninth International Symposium on, pages 346-353, 2001.

[41] J. Mirkovic. A Taxonomy of DDoS Attack and DDoS Defense Mechanisms. ACM SIGCOMM Computer Communication Review, 34(2):39-53, 2004.

[42] K. Park and H. Lee. On the Effectiveness of Route-Based Packet Filtering for Distributed DoS Attack Prevention in Power-Law Internets. In Proceedings of the 2001 conference on Applications, technologies, architectures, and protocols for computer communications, pages 15-26. ACM New York, NY, USA, 2001.

[43] J. Postel. Internet Protocol. Internet Request for Comments RFC 791, Sept. 1981.

[44] J. Rajahalme, A. Conta, B.E. Carpenter, and S.E. Deering. IPv6 Flow Label Specification. Internet Request for Comments RFC 3697.

[45] T. Rehman. Implementation of Radix Tree, March 2008. [Online]. Available: http://code.google.com/p/radixtree/, (Jan. 2009).

[46] Y. Rekhter, T. Li, and S. Hares. A Border Gateway Protocol 4 (BGP-4). Internet Request for Comments RFC 4271, 2006.

[47] R. Rivest. The MD5 Message-Digest Algorithm. Internet Request for Comments RFC 1321, 1992. 
[48] S. Sangli, D. Tappan, and Y. Rekhter. BGP Extended Communities Attribute. Internet Request for Comments RFC 4360, 2006.

[49] S. Savage, D. Wetherall, A. Karlin, and T. Anderson. Practical Network Support for IP Traceback. In SIGCOMM '00: Proceedings of the conference on Applications, Technologies, Architectures, and Protocols for Computer Communication, pages 295-306, New York, NY, USA, 2000. ACM.

[50] AC Snoeren, C. Partridge, LA Sanchez, CE Jones, F. Tchakountio, B. Schwartz, ST Kent, and WT Strayer. Single-Packet IP Traceback. Networking, IEEE/ACM Transactions on, 10(6):721-734, 2002.

[51] D.X. Song and A. Perrig. Advanced and Authenticated Marking Schemes for IP Traceback. In INFOCOM 2001. Twentieth Annual Joint Conference of the IEEE Computer and Communications Societies. Proceedings. IEEE, pages 878$886,2001$.

[52] J. Stewart, T. Bates, R. Chandra, and E. Chen. Using a Dedicated AS for Sites Homed to a Single Provider. Internet Request for Comments RFC 2270, 1998.

[53] WT Strayer, CE Jones, F. Tchakountio, and RR Hain. SPIE-IPv6: Single IPv6 Packet Traceback. In Local Computer Networks, 2004. 29th Annual IEEE International Conference on, pages 118-125, 2004. 
[54] T. Wan, E. Kranakis, and P. van Oorschot. Pretty Secure BGP (psBGP). In In The 12th Annual Network and Distributed System Security Symposium (NDSS05), 2005.

[55] T. Wan, P. VAN Oorschot, and E. Kranakis. A Selective Introduction to Border Gateway Protocol (BGP) Security Issues. In Proc. of NATO Advanced Studies Institute on Network Security and Intrusion Detection, 2007.

[56] R. White. Securing BGP through secure origin BGP (soBGP). The Internet Protocol Journal, 6(3):15-22, 2003.

[57] J. Winick and S. Jamin. Inet-3.0: Internet topology generator. Technical report, 2002.

[58] A. Yaar, A. Perrig, and D. Song. FIT: Fast Internet Traceback. In INFOCOM 2005. 24th Annual Joint Conference of the IEEE Computer and Communications Societies. Proceedings IEEE, pages 1395-1406, 2005.

[59] A. Yaar, A. Perrig, and D. Song. StackPi: New Packet Marking and Filtering Mechanisms for DDoS and IP Spoofing Defense. Selected Areas in Communications, IEEE Journal on, 24(10):1853-1863, 2006. 